\title{
Classification of ST segment in ECG signals based on cross correlated supervised data
}

\author{
Md. Harun-Ar-Rashid' ${ }^{1}$ Golam Mahmud ${ }^{1}$. Mohammad Motiur Rahman ${ }^{2}$. A. S. M. Delowar Hossain ${ }^{2}$
}

Received: 7 December 2019 / Accepted: 10 June 2020 / Published online: 15 June 2020

(c) Springer Nature Switzerland AG 2020

\begin{abstract}
This paper describes an automated selection of the ST segment in 12 leads electrocardiogram (ECG) as well as its classification based on cross correlation. Our proposed method classifies five categories of ST segment which are (a) Up slop (b) Down slop (c) Horizontal (Normal) (d) Concave (e) Convex using cross correlation process. We compare the main ECG (patient ECG) ST segment with the above-mentioned reference ST segments. In this work we have used MIT-BIH ST change database and European ST-T change database where every database contains minimum 30 min and maximum 1-h episode. Our method contains the following steps (1) Filtering ECG signal and Detrending it (2) R peak and S peak detection (3) Starting and ending point detection of ST segment (4) Comparing with ST segment supervised data (5) Classifying the ST segment. We have used total 1,34,879 beats where 58,331 beats from MIT-BIH ST change database and 74,609 beats from European ST-T change database. We have correctly selected total 126,608 ST segments. ST segment classification accuracy is $88.20 \%$ for MIT-BIH ST change database and $96.18 \%$ for European ST-T change database. The method confirms satisfactory performance with an overall accuracy of $92.1 \%$ which is helpful to the detection of major heart diseases like myocardial ischemia.
\end{abstract}

Keywords Myocardial ischemia $\cdot$ Detrended electrocardiogram (ECG) $\cdot$ Cross correlations $\cdot$ ST segment ramification

\section{Introduction}

It is important to extract the features of ECG signals to find the weakness of the heart of a patient. Electrocardiogram contains different types of wave such as $\mathrm{P}, \mathrm{Q}, \mathrm{R}, \mathrm{S}, \mathrm{T}, \mathrm{U}$ wave (Fig. 1). Most of the time $U$ waves are hidden. $Q, R, S$ waves are called QRS Complex. Due to heart rhythm, the shape of ECG signal changes over time. At the end of $S$ wave J point starts, this detection is important for detecting myocardial ischemia. Most of the studies focus on $P, R$ and $T$ wave detection and T wave alternation [1].

It is not easy for physicians to extract features of ECG from visual perception. So, developing an algorithm on ECG signal for finding required features will be more helpful for physicians. Reduction of blood flow to our heart for myocardial ischemia prevents the supply of enough oxygen. This reduced blood flow sometimes partially blocks our heart arteries. This myocardial ischemia may also be called cardiac ischemia which can damage our heart muscle by decreasing the ability of pump.

Myocardial ischemia is identified by monitoring end point of S wave to start point of T wave. This part is a segment of ECG signal which is called ST segment. Our proposed method focuses on this ST segment changes and classifies it based on cross correlation method. Naturally ST segment is isoelectric with slightly slanted upwards form contained in the middle of ventricular depolarization and repolarization (Fig. 2).

\footnotetext{
$\triangle$ Mohammad Motiur Rahman, mm73rahman@gmail.com | ${ }^{1}$ Computer Science and Engineering, Mawlana Bhashani Science and Technology University, Tangail 1902, Bangladesh. ${ }^{2}$ Faculty Member, Computer Science and Engineering, Mawlana Bhashani Science and Technology University, Tangail 1902, Bangladesh.
} 


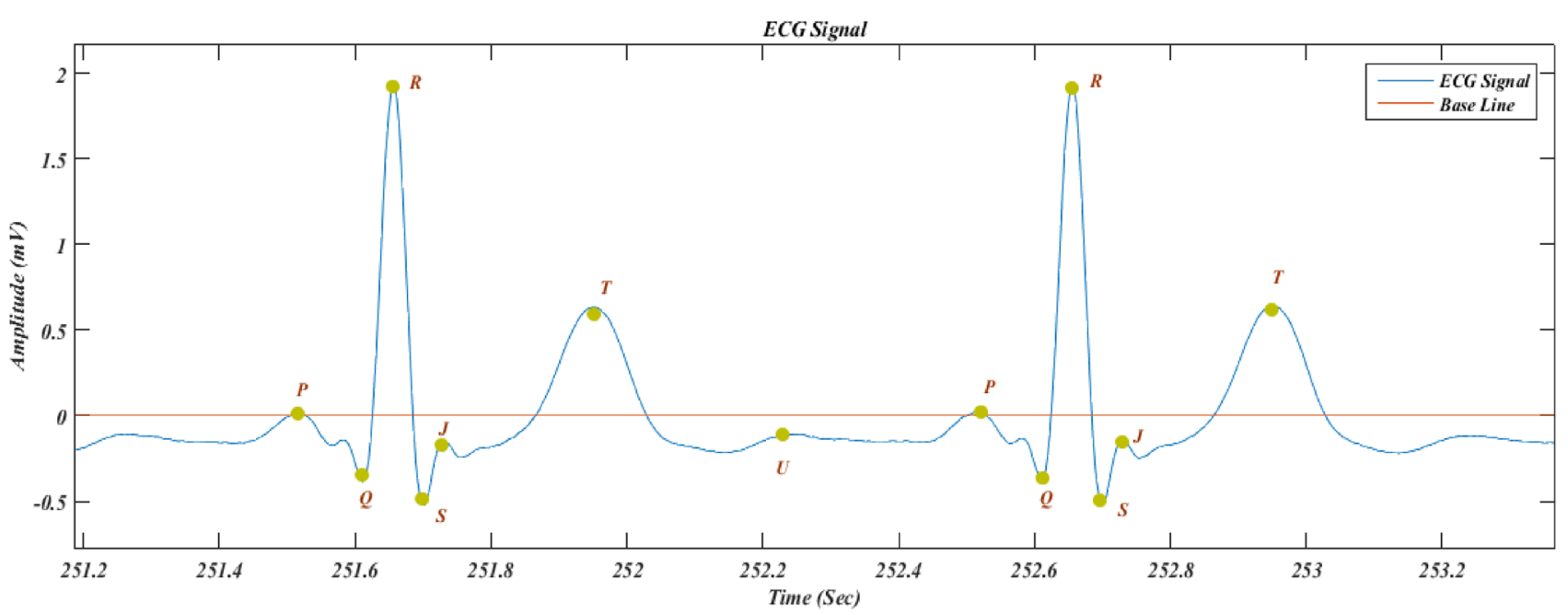

Fig. 1 ECG signal with $\mathrm{P}, \mathrm{QRS}$ complex, T and U wave

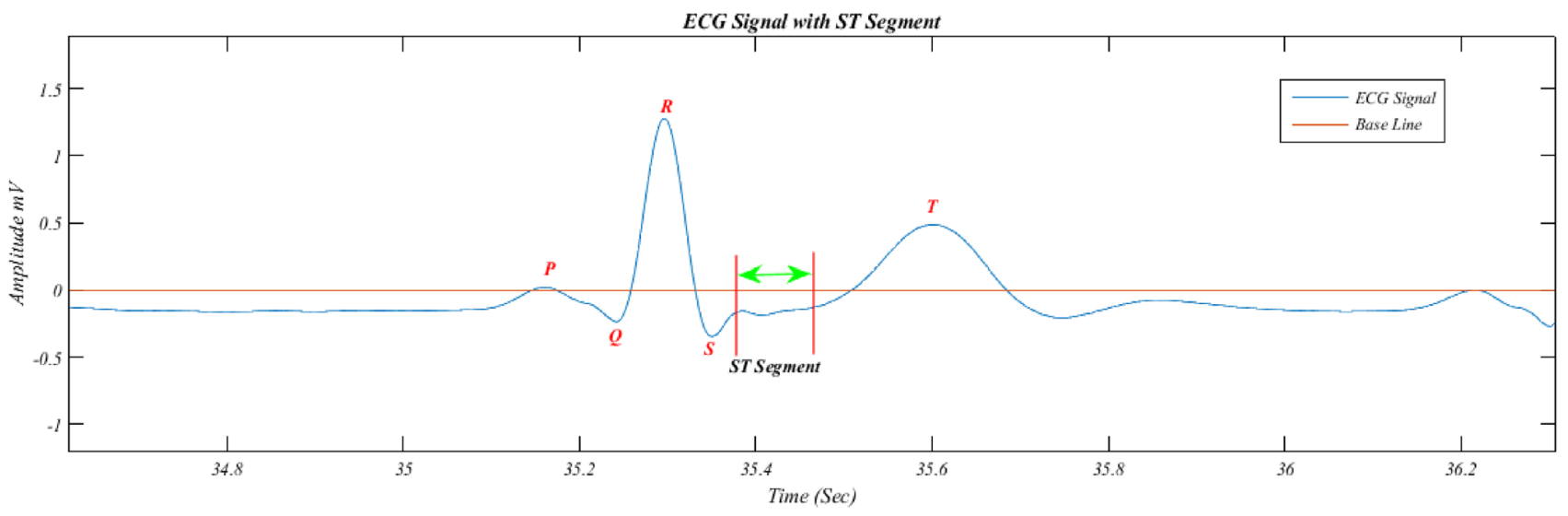

Fig. 2 ECG signal with ST-segment

Most of the available methods are developed based on time domain or sloping based for detecting ST segment. Some methods also focus on support vector machine (SVM) [2]. Different kinds of algorithms monitor the ECG signal. Some of them provide normality and abnormalities of regular ECG sinus rhythm. A new approach is proposed to detect normal and abnormal ECG signal but does not give detailed information [3]. Another method [4] provides ECG arrhythmia classification based on discrete wavelet transform (DWT). Classification task is performed by MultiLayer Perceptron (MLP) neural network and it shows two arrhythmias Paced Beat (PB) and Atrial Premature Beat (APB). Two step classification process is described in the work. ST-T elevation and depression are classified from the average pattern of ECG. Paper [5] provides the variation of ST-T complex changes. A detector, which finds the changes of ST-T complex and average pattern of ST segment amplitude. Method [6] monitors the ST segment elevation and depression in different lead for occlusion of coronary arteries. It monitors the ST Segment on 45 patients of 19 leads with the sensitivity or specificity. They also have measured the elevation of ST segment based on their amplitude value ( $\mathrm{mV}$ ). Paper [7] shows non-ST-segment elevation, myocardial infraction, unstable angina detection. They also have developed the standard protocol for selecting ST segment and ST-segment elevations for new-born. Diffusion of ST segment provides for only depression of ST segment. Significance of ST segment for general people is done and compared with hypothermia patients in [8]. Fatal ST segment is detected from fatal heart rate monitoring in [8]. They have also compared the ST segment with normal patient and has given the additional information of fatal ST segment [9]. A process is developed for detecting ST segment and classified six major groups, i.e. Normal, Ventricular, Atrial, Fusion, Right Bundle, and Left Bundle Branch Block beats by Discrete Wavelet Transformation (DWT), 
Windowing Technique and Slope Detection process [10]. ST segment abnormalities detection based on Signal Block Averaging Technique (SBAT) is also developed in [11]. They have developed an algorithm and applied SBAT for each ECG episode. Exact point of ST segment is end point of QRS complex and start point of T wave. Most of the time ST segment has monitored as depression and elevation type also traced abnormal ST Segment in ECG tracing [12].

Our proposed method automatically detects ST segment and classifies those ST segment into five categories using cross correlation based on supervised data.

The remaining part of this paper is structured as follows: Sect. 2 describes the proposed methodology as well as ST segment detection and classification process. The experimental results are analyzed in Sect. 3. Finally, discussion and conclusions are drawn in Sects. 4 and 5 respectively.

\section{Methodology}

Our proposed method consists of following steps shown in (Fig. 3). This figure describes overall summary of our proposed system. Every section is important for monitoring the ST segment.

\subsection{ECG database}

Here, we use MIT-BIH ST change database as a training data and European ST-T change database as a testing data. Training database contains a total of Twenty-eight ECG signals and the testing database contains a total of Nineteen ECG signals.

Training database is used to train our proposed method. Testing database is used to verify our proposed method. MIT-BIH ST change database is used for training where every ECG signal has its individual name from 301 to $327 \mathrm{~m}$ and sampling frequency is $360 \mathrm{~Hz}$. On the other hand, the European ST-T change database is used for testing where every ECG signal has its individual name from e0103 to e0606m and the sampling frequency is $250 \mathrm{~Hz}$ [13].

Training and testing databases are separated from their name and sampling frequency.

\subsection{Data pre-processing}

\subsubsection{Filtering data}

There are several types of denoising techniques developed for smoothing and denoising the ECG signal [14-16] such as denoise using Intrinsic Mode Function based on EMD (Empirical Mode Decomposition) for detecting QRS complex. Here we use the MIT-BIH database and European ST-T database which is sampled at $360 \mathrm{~Hz}$ and $250 \mathrm{~Hz}$, respectively. We use FIR bandpass filter of $0.05-45 \mathrm{~Hz}$ with 100 order and Savitzky-Golay smoothing filtering technique. Every ECG signal contains 30 min or above episode.

\subsubsection{Detrending data}

After denoising ECG signal contains a trend. Every ECG signal may contain linear and nonlinear trends. For detrending, we use polynomial Eq. (1) of degree $n$. Then we have calculated mean, the standard deviation of time series signal. Figures 4 and 5 are showing before detrending and after detrending operations respectively. After detrending, we can easily identify the baseline.

$P(x)=p_{1} x^{n}+p_{2} x^{n-1}+\cdots+p_{n} x+p_{n+1}$

$\hat{x}=\left(x-\mu_{1}\right) / \mu_{2}$

Here $p_{1}, p_{2} \ldots \ldots p_{n}$ are the coefficients of the polynomial, $x$ is the independent variable of signal in time domain. $\mu_{1}$ is the mean of $x$ and $\mu_{2}$ is standard deviation of $x$.

\section{3 $R$ wave and $S$ wave detection}

For detecting $\mathrm{R}$ wave peak value, we use following function.

$f(d, h)=\{n, e(n)$ when $\Delta n \geq d$ and $e(n) \geq h$

where $h$ parameter denotes minimum peak height from the base line where amplitude value $m V=0$ and $d$ denotes minimum peak distance from previous $\mathrm{R}$ to $\mathrm{R}$ peak interval, $\Delta n$ is the minimum peak distance after selecting peak. For every ECG signal $\mathrm{n}$ is the time value and $e(n)$ is the amplitude value at the time $n$. Above function provides local maxima in minimum interval which is the main $R$ peak value. Here local maxima mean maximum amplitude value between the time interval $\Delta n$ [17].

For detecting $\mathrm{S}$ wave peak value, we use following function.

$f^{\prime}\left(d^{\prime}, h^{\prime}\right)=\left\{n, e^{\prime}(n)\right.$ when $\Delta n \geq d^{\prime}$ and $e(n) \geq h^{\prime}$

where $\boldsymbol{h}^{\prime}$ parameter denotes minimum peak height from the baseline where amplitude value $m V=0$ and $\boldsymbol{d}^{\prime}$ denotes minimum peak distance from previous $S$ to $S$ peak interval. $\Delta \boldsymbol{n}$ is the minimum peak interval after selecting peak.

After using Eqs. (3) and (4) we have found the R wave and $S$ wave peak value. Figure 6 shows $R$ wave and $S$ wave detection where $S$ wave is marked with ' $\square$ ' $R$ wave is marked with' ${ }^{\prime}$ '. 


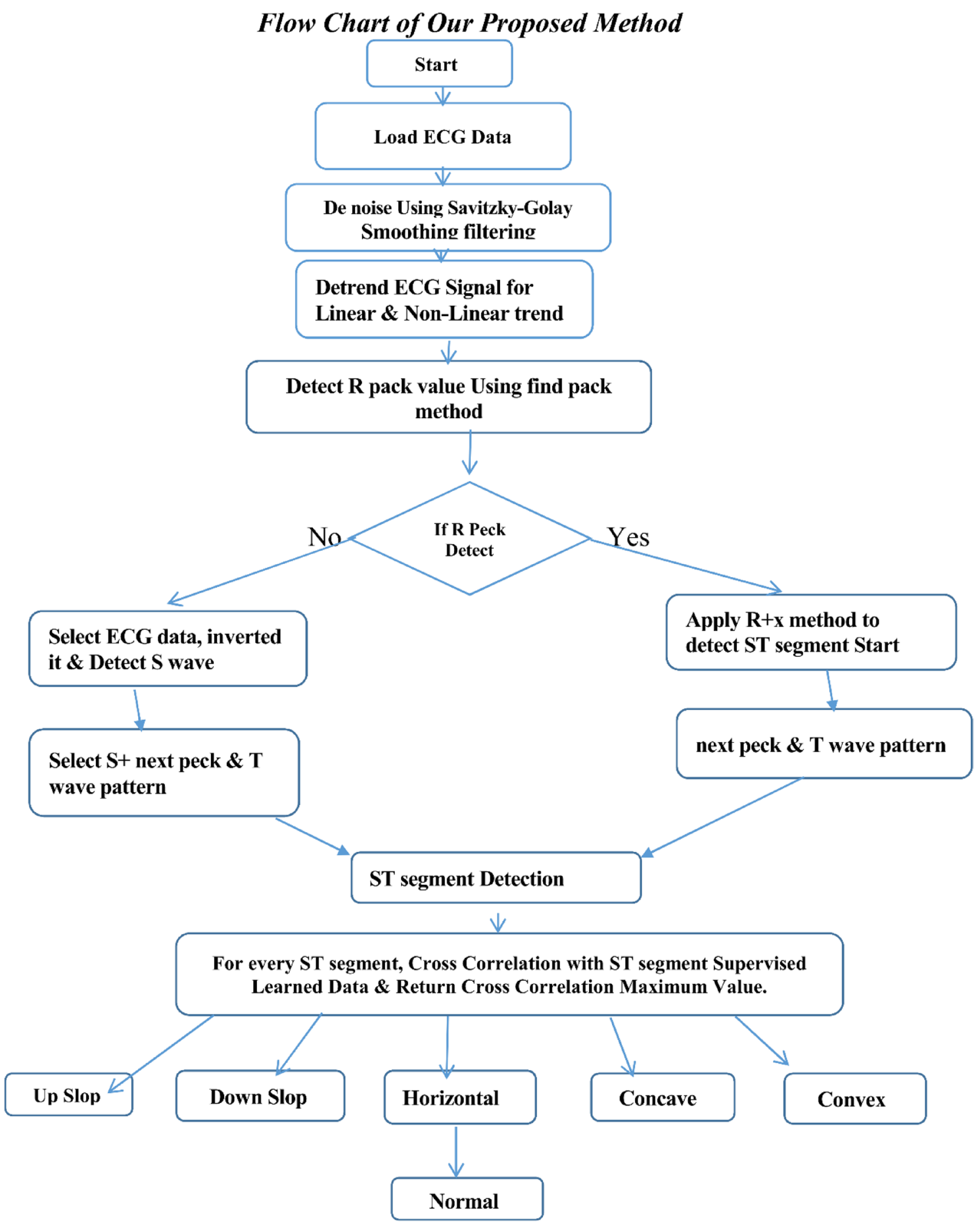

Fig. 3 Flow chart of our proposed method

\subsection{Start and endpoint detection of ST segment}

ST segment can be defined by ending point of $S$ wave and start point of T wave $[18,19]$.

\subsubsection{J point detection}

$J$ point is the indicator of the end of ventricular depolarization [20]. It is the start point of ST segment. It can 


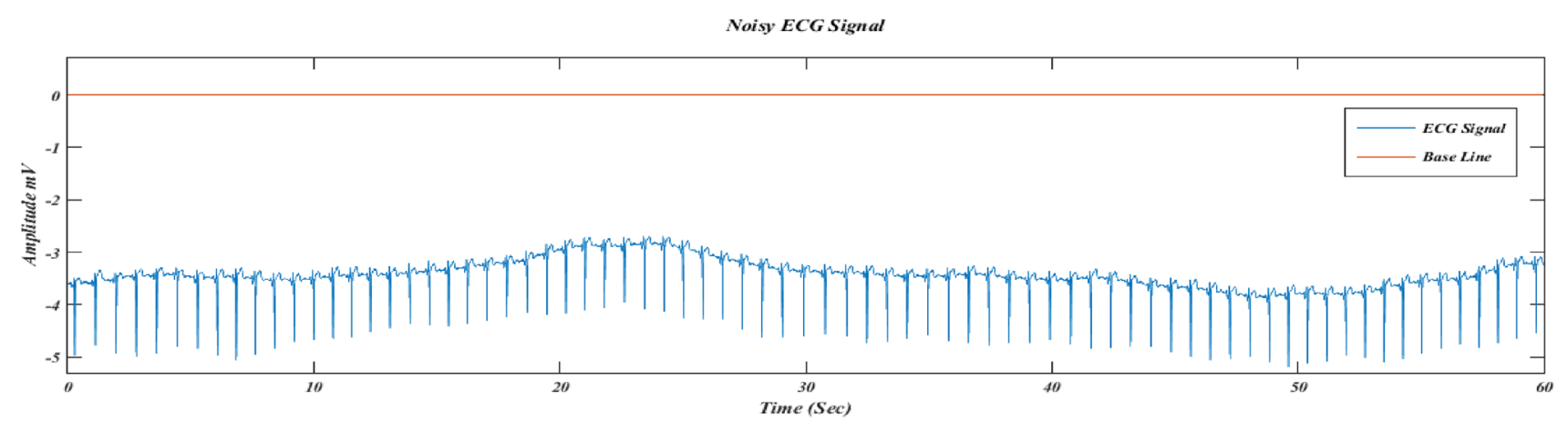

Fig. 4 ECG signal with linear and non-linear trend

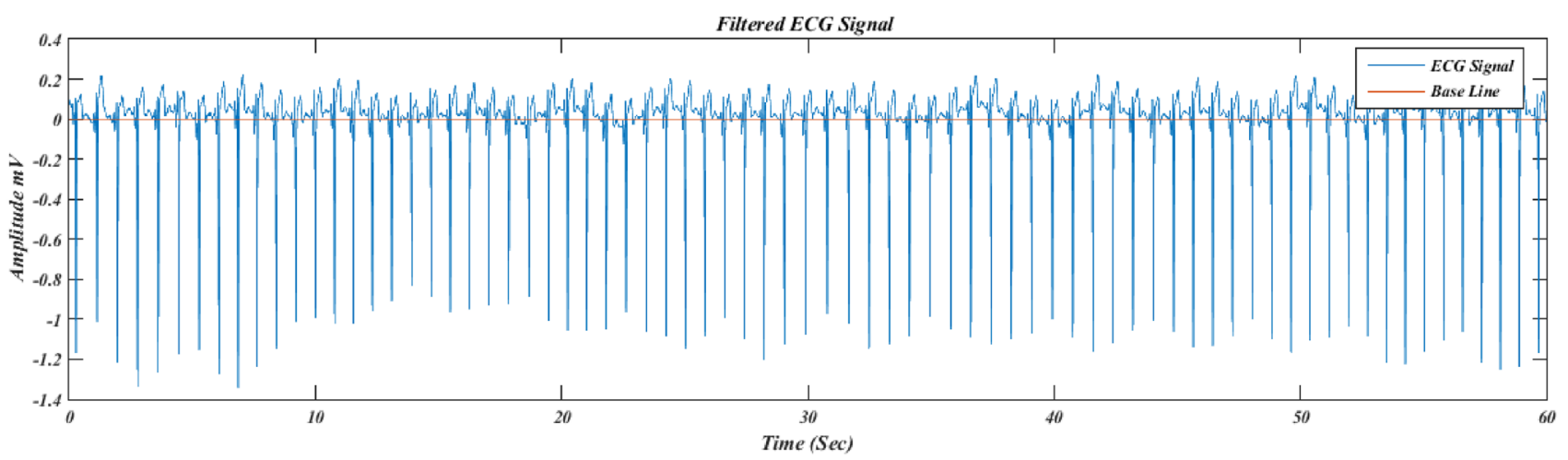

Fig. 5 ECG signal after removing linear and non-linear trend

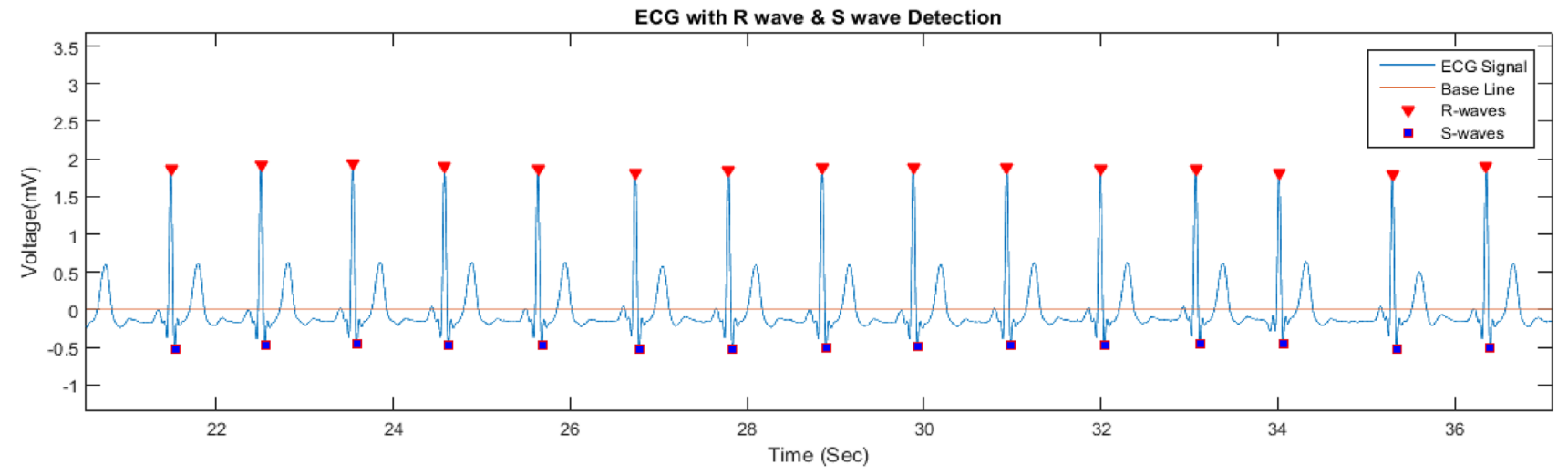

Fig. $6 \mathrm{R}$ wave and S wave detection in ECG

be identified on different R-R interval. Sometimes it provides pattern of normality and abnormality of ST segment. Different J point can be detected on different leads [20]. We have applied $\mathrm{R}+\mathrm{x}$ method to detect it [21]. Here $R$ is the highest peak position (getting time domain value) in $\mathrm{R}$ wave and $x$ is also time domain value. After getting $\mathrm{R}$ wave highest peak position then $x$ is added with that position to get J point. Here $x$ depends on the RR interval or SS interval. Because RR interval or SS interval duration is not fixed in ECG signal. If RR or SS interval increase, then position of J point position also increases.

For $\mathrm{R}$ based we use 
$\left\{\begin{array}{c}\text { if } \Delta R<160 \text { then } x=10 \\ \text { if } 160 \leq \Delta R<180 \text { then } x=12 \\ \text { if } 180 \leq \Delta R \leq 270 \text { then } x=14 \\ \text { if } 270<\Delta R \leq 390 \text { then } x=16 \\ \text { if } \Delta R>390 \text { then } x=20\end{array}\right\}$

For $\mathrm{S}$ based we use

$$
\left\{\begin{array}{c}
\text { if } \Delta S<160 \text { then } x=2 \\
\text { if } 160 \leq \Delta S \leq 180 \text { then } x=4 \\
\text { if } 180<\Delta S \leq 270 \text { then } x=6 \\
\text { if } 270<\Delta S \leq 360 \text { then } x=8 \\
\text { if } \Delta S>360 \text { then } x=10
\end{array}\right\}
$$

Here $\Delta R$ is the RR interval and $\Delta S$ is the SS interval. That's mean if first $R$ wave height peak found in $R_{1}$ time and second $R$ wave height peak found in $R_{2}$ time. Then $\Delta R$ is the difference between two $R$ waves that is $\Delta R=R_{2}-R_{1}$. $R R$ interval detects $R$ peak and $S S$ interval detects $S$ peak. Here $x$ is the time domain value. After detecting $\mathrm{R}$ or $\mathrm{S}$ waves highest peak position $x$ is added to that position to get the start point of ST segment or $J$ point. Sometimes some lead contains less magnitude of $R$ peak. They contain dissimi$\operatorname{lar} R$ peak value [22]. In these cases, missing of $R$ wave for example Lead $V 1$ we use $S$ wave and then apply the above method. That means if $R$ peak is not detected then $S$ based process is used. If $\mathrm{R}$ or $\mathrm{S}$ wave is not detected, then above method will not work.

\subsection{ST segment endpoint detection}

Endpoint of ST segment is the start point of T wave. We calculate the angle from ST segment start point to T wave peak value. Though $T$ wave detection method is developed [23-26] but we have used slope-based process.

$$
\begin{aligned}
\tan \theta & =\frac{y_{2}-y_{1}}{x_{2}-x_{1}} \\
& \Rightarrow \theta=\tan ^{-1} \frac{y_{2}-y_{1}}{x_{2}-x_{1}}
\end{aligned}
$$

We calculate the angle using Eq. (7) where $x_{1}, y_{1}$ is the start point and $x_{2}, y_{2}$ is the end point.

From the angle value, we can see (Fig. 7) $\theta_{1}<\theta_{2}<\theta_{3}<\theta_{4}<\theta_{k}<\theta_{n}$ that means when angle goes closer to perpendicular it increases. We applied the above process for detecting $T$ wave start point.

From the angle value, we calculate the maximum angle then select the start point of T wave (Fig. 8).

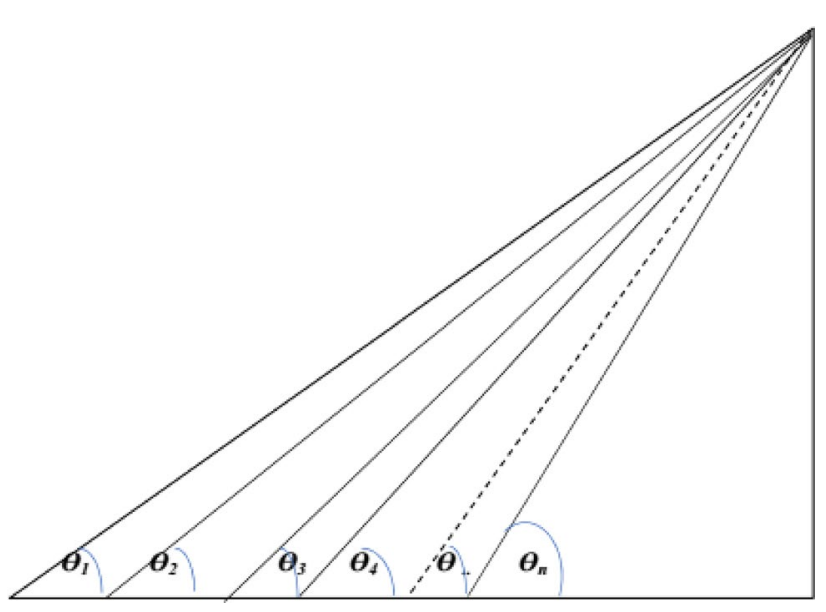

Fig. 7 Right triangle with different hypotenuse

\subsection{ST segment separation and dissimilarities find out}

For every ST segment, we have applied supervised learning. Firstly, we select some ST segments from our European ST-T change and MIT-BIH ST change database. This reference ST segment is treated as supervised ST signal. We selected 5 categories of supervised ST signal. Each ST category contains 2 or 3 ST segments. Then we compare this selected reference ST segment with our main ECG ST segment using Cross Correlation (Fig. 9).

\subsubsection{Cross Correlation Applied}

Cross correlation is useful for estimating the similarities between two signals corresponding to time domains. Here, we take two signal parts, one is the main ST Segment that should be classified which is denoted by $X_{k}(t)$ and the other is our supervised signal $Y_{j}(t)$ which is compared with $X_{k}(t)$ signal. Where $k$ is the $k$ th number of ST segment of main ECG and $j$ is $j$ th supervised signal.

The cross-correlation $r$ can be defined as

$$
r=\frac{\sum[(X(i)-m x) *(Y((i-d)-m y)]}{\sqrt{\sum((X(i)-m x))^{2}} \sqrt{\sum(Y(i-d)-m y)^{2}}}
$$

where $m x$ and $m y$ are the means of the corresponding series, $d$ is the time delay and $d=0$ to $N-1$.

$\boldsymbol{r}(\boldsymbol{d})=\frac{\sum[(\boldsymbol{X}(\boldsymbol{i})-\boldsymbol{m} \mathbf{x}) *(\boldsymbol{Y}((\boldsymbol{i}-\boldsymbol{d})-\boldsymbol{m} \boldsymbol{y})]}{\sqrt{\sum((\boldsymbol{X}(\boldsymbol{i})-\boldsymbol{m} \boldsymbol{x}))^{2}} \sqrt{\sum(\boldsymbol{Y}(\boldsymbol{i}-\boldsymbol{d})-\boldsymbol{m y})^{2}}}$

The range of $r(d)$ is $-1<=r(d)<=1$, where 1 indicating maximum correlation and 0 indicating no correlation. 


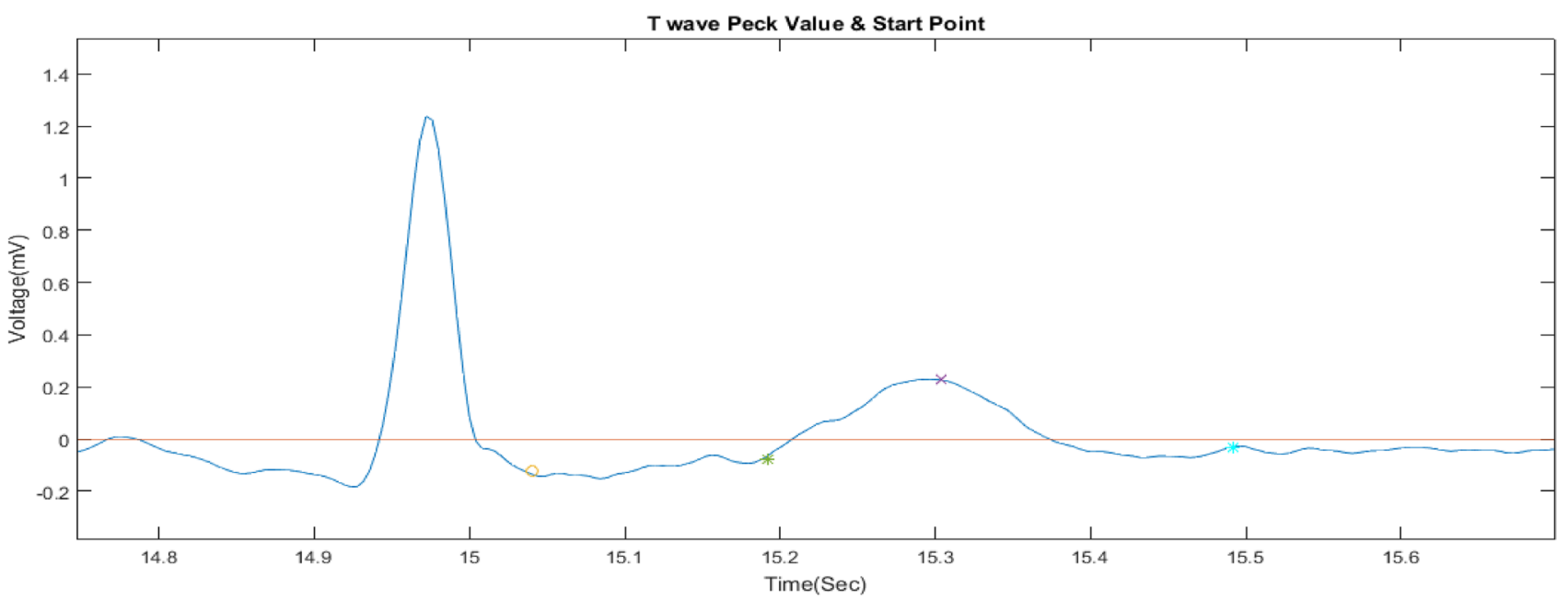

Fig. 8 ST segment endpoint detection and T wave detection in ECG
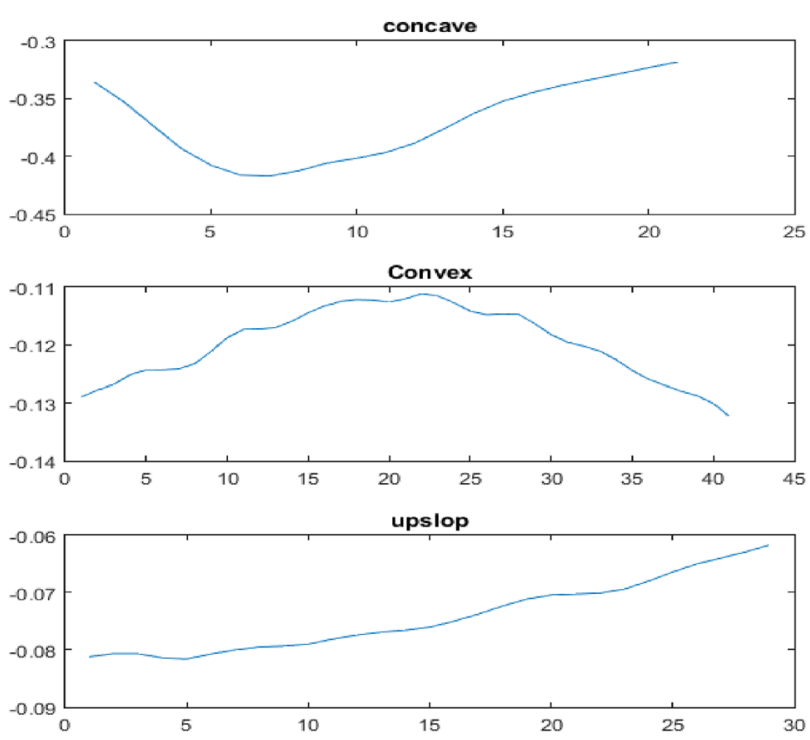

Fig. 9 Five kinds of supervised data

Here we have applied cross correlation between $X_{k}(t)$ and $\sum_{j=0}^{M} \boldsymbol{Y}_{j}(\boldsymbol{t})$ where $M$ is total number of supervised signals. For every $j$ value, cross correlation between $Y_{j}(t)$ and $X_{k}(t)$ returns $r_{j}(d)$ where $r(d)$ is $-1<=r(d)<=1$. For all $r_{j}(d)$ value we find out the maximum $r(d)$ value. If $k$ th number of $r(d)$ is maximum, then we can say $X_{k}(t)$ is cross correlated with $k$ th supervised signal. If $k$ th supervised signal is upslope or downslope, then we can say $X_{k}(t)$ is upslope or downslope (Fig. 10) and (Fig. 11).
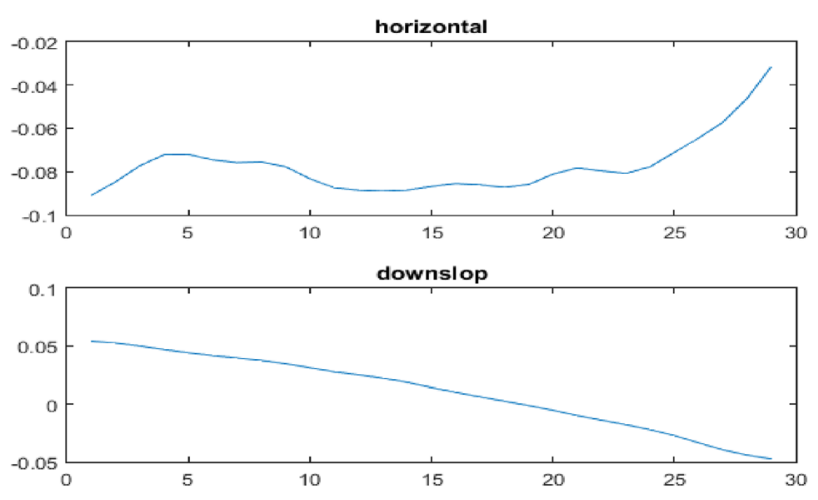

\section{Result}

We have used European ST-T change database and MITBIH ST change database total 1,34,879 beat. Nineteen ECG signals from European ST-T and twenty-eight ECG signals from MIT-BIH ST change database are selected. Every database contains minimum $30 \mathrm{~min}$ to maximum $1 \mathrm{~h} 6$-min episode. 

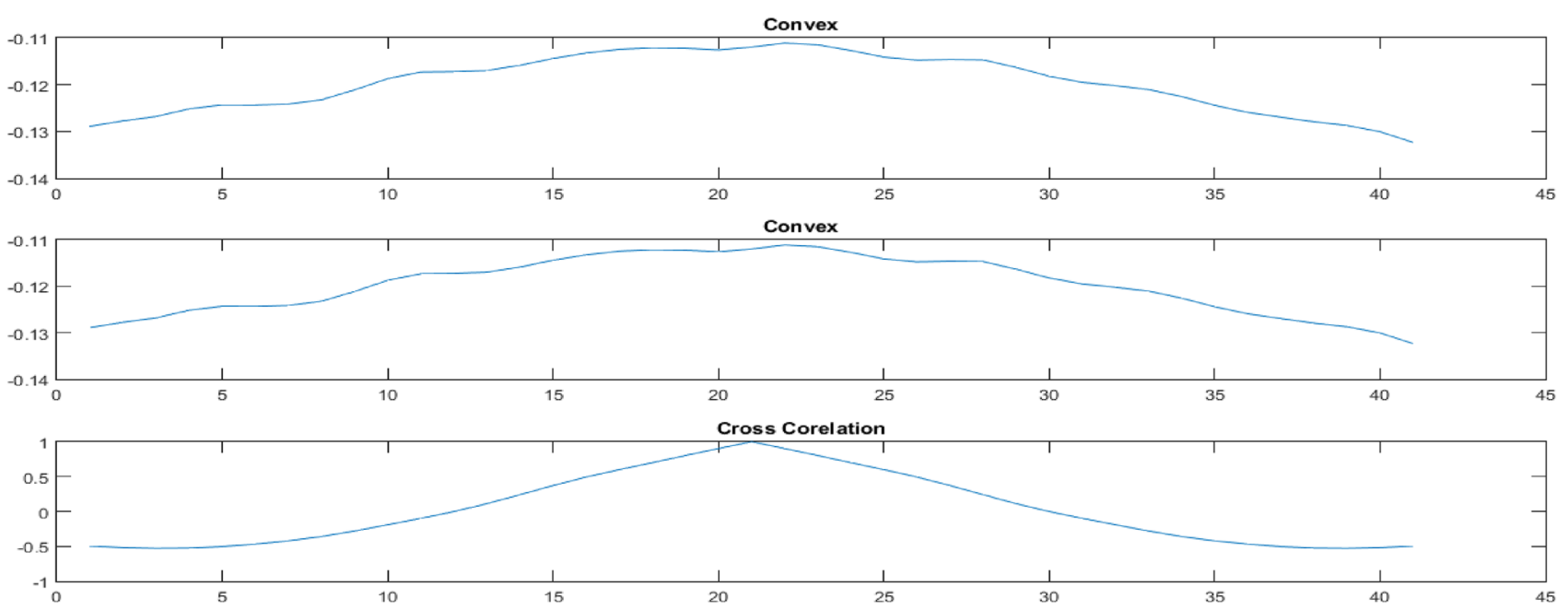

Fig. 10 Cross correlation between similar signal
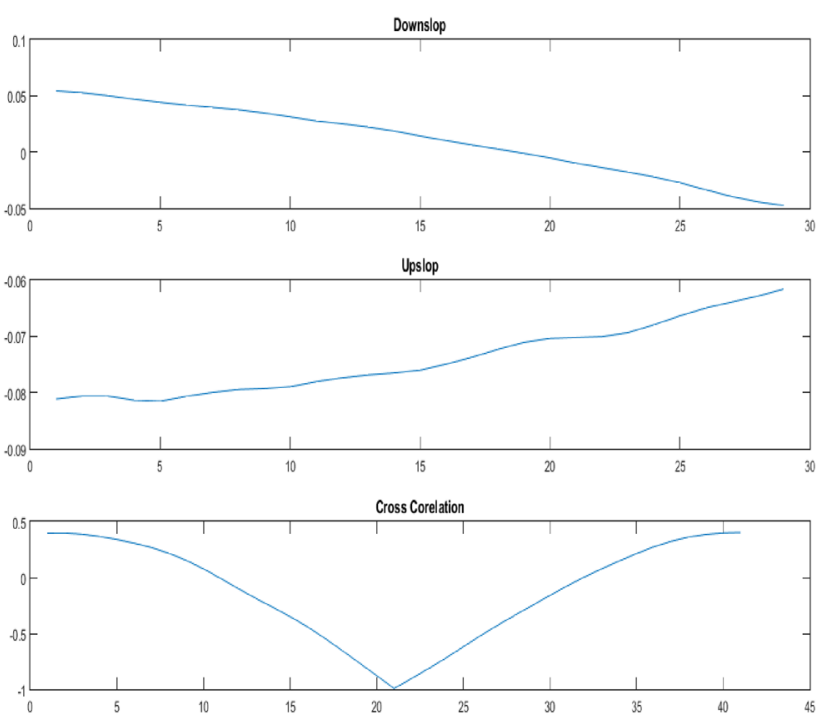

Fig. 11 Cross correlation between negatively correlated signal

ST segment start point means the J point. In our proposed method J point detection depends on $\mathrm{R}$ wave peak or S wave peak. In this case if $\mathrm{R}$ wave select correctly or $S$ wave select correctly then $J$ point detection is more accurate [Eqs. (5) and (6)]. If $R$ wave or $S$ wave detection is wrong that time J point position is also wrong. In this case we marked $\mathrm{R}$ wave with ' $\mathrm{w}$ ' symbol, $\mathrm{S}$ wave with ' $\boldsymbol{\nabla}$ ' symbol and J point marked with 'o' symbol. After marking with symbol, we monitored each ECG signal and noted down which point is correctly selected or not. Sometimes $\mathrm{R}$ wave or $\mathrm{S}$ wave detects wrongly that time J point select wrongly. That type of case ST segment selects wrongly.

ST segment end point means T wave start point. In this case, we applied the $T$ wave pattern detection process
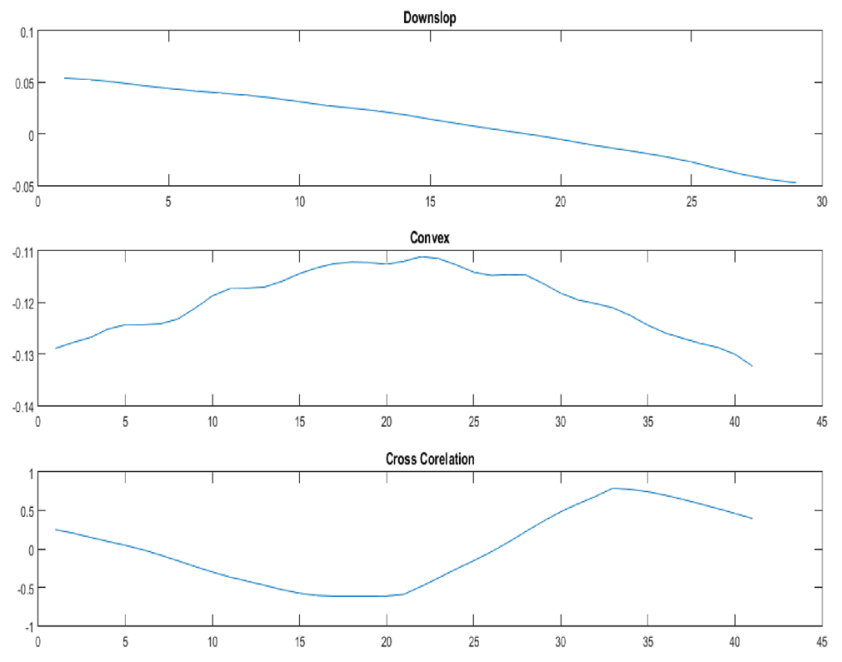

using the right triangle hypotonus system [Eq. (7)]. In $T$ wave pattern we find out $T$ wave start point which is marked with ' $x$ ' symbol.

Using above process ST segment start point marked with 'o' symbol and end point marked with ' $x$ '. In every ECG signal ST segment is monitored.

In our selection process ST segment start point depends on $\mathrm{R}$ or $\mathrm{S}$ wave and end point depends on T wave. If ST segment start point marked position varies $\pm 3 \mathrm{~ms}$ and end point marked position varies $\pm 6 \mathrm{~ms}$ and their time duration varies from 0.005 to $0.150 \mathrm{~s}$, then it is treated as correctly selected ST segment otherwise it is treated as wrongly selected ST segment. If any of the start point or end point selects wrongly such as marking position is not 
between $\mathrm{S}$ and T wave interval or start point position is inside the interval and end point position is outside the interval or any of them then that type of case ST segment treated as wrongly selected ST segment.

Only correctly selected ST segment is classified. We classified ST segment into 5 categories which are concave, convex, upslope, downslope and horizontal. Another study [27] also classified ST segment based on ST segment start point amplitude value $(\mathrm{mV})$, end point amplitude value $(\mathrm{mV})$ and average amplitude value $(\mathrm{mV})$. If ST segment average amplitude value is greater than ST segment start and end point amplitude value, then this type of ST segment is treated as convex type ST segment. If ST segment average amplitude value is less than ST segment start and end point amplitude value, then this type of ST segment is treated as concave type ST segment. If ST segment average amplitude value is between the ST segment start and end point amplitude value and end point amplitude value is greater than start point amplitude value, then this type of ST segment is treated as upslope type ST segment. If ST segment average amplitude value is between the ST segment start and end point amplitude value and end point amplitude value is less than start point amplitude value, then this type of ST segment is treated as upslope type ST segment. If ST segment average amplitude value is approximate to the ST segment start and end point amplitude value, then this type of ST segment is treated as horizontal type ST segment.

Using the above rules, we selected 5 categorized of supervised data (Fig. 9). Our tested ST segment type is annotated using both rules. In our tested ST segment, we applied the cross-correlation process [Eqs. (8), (9)]. Our tested ST segment type is verified by average amplitude value [27]. Our tested ST segments type is treated as correctly selected type when it is satisfied both average amplitude value rules [27] and our proposed rule (cross-correlation).

\subsection{Classification result}

ST segment is classified based on cross correlation. Figures $12,13,14,15$ and 16 shows classification result of ST segment. Here Figs. $12 \mathrm{~b}$ and $12 \mathrm{c}$ are the zoomed version of Fig. 12a. Duration of ST segment varies from 0.005 to $0.150 \mathrm{~s}(5-150 \mathrm{~ms})$.

We classify the result based on correctly selected ST segment and Wrongly selected ST segment. Total correctly selected ST segment is denoted by $T_{s t}$ and wrongly selected ST segment is denoted by $F_{s t}$. Now we have applied the formula $\frac{\sum \text { Tst }_{i}}{\sum \mathrm{Tst}_{i}^{+} \sum \mathrm{Fst}_{i}}$ for calculating the total accuracy. Sometimes same ECG episode contains different types of ST segment as Horizontal and Upslope. Our method selects all segments and ramifies them. Total number of correctly identified ST segment for each shape is divided by the total number of shapes existed in the signal, as find the accuracy.

From the Tables 1 and 2 we see ST segment accuracies are $96.79 \%$ and $98.96 \%$. Classification accuracy for European ST-T change database are $95.58 \%$ for concave, $95.92 \%$ for convex, $97.86 \%$ for upslope, $95.18 \%$ for downslope, $96.36 \%$ for horizontal. For MIT-BIH ST change database Horizontal accuracy is less than $70 \%$ because of at the same signal it contains horizontal and convex shape. Some horizontal shape contains upslope or other up down shape. Cross Correlation process can't easily identify this. But in European ST-T change database it provides more accuracy because its database contains smoother horizontal shape, Cross Correlation process identifies this shape 96.36\% accurately which is more than MIT-BIH ST change database.

\section{Discussion}

Previous study [27] shows a rule-based method for ST segment classification. It measures the amplitude value of ST segment start and endpoint. If average amplitude value is greater than start point then it is considered as elevation, otherwise it is considered as depression. It is one kind of baseline-based ST Segment classification. Sometimes some horizontal shapes are found in ST segment depression type. Average accuracy is $90.1 \%$ for testing data [27]. Another method shows ST segment upslope and downslope type over 31 patients [28]. This study [28] selects the ST segment from J point to $60 \mathrm{~ms}$ and has classified the ST Segment based on their amplitude value. Curvature Scale Space based ST segment classification is also developed [29]. This study [29] shows $91.60 \%$ accuracy for small amount of ECG data. It eliminates the endpoint detection of the ST segment. Philips Medical Systems [30] shows ST segment monitoring process. They also show the ST segment selecting process and selected the ST segment from the voltage difference between the value at a point 60 or $80 \mathrm{~ms}$ after the J-point and the isoelectric baseline [30]. This study [30] show only four types of ST segment which are normal, abnormal, elevation and depression types [30]. Although above study have achieved good result and classification but most of the study applied on small duration of ECG data, small amount of testing and training data with over all accuracy $90 \%$.

Our present study has proposed detection and classification of ST Segment. In this study MIT-BIH ST change database is used for training. Here we have used both $\mathrm{R}, \mathrm{S}$ and T wave pattern for detecting ST Segment. For detecting $J$ point we have used both $R$ wave pattern and $S$ wave 


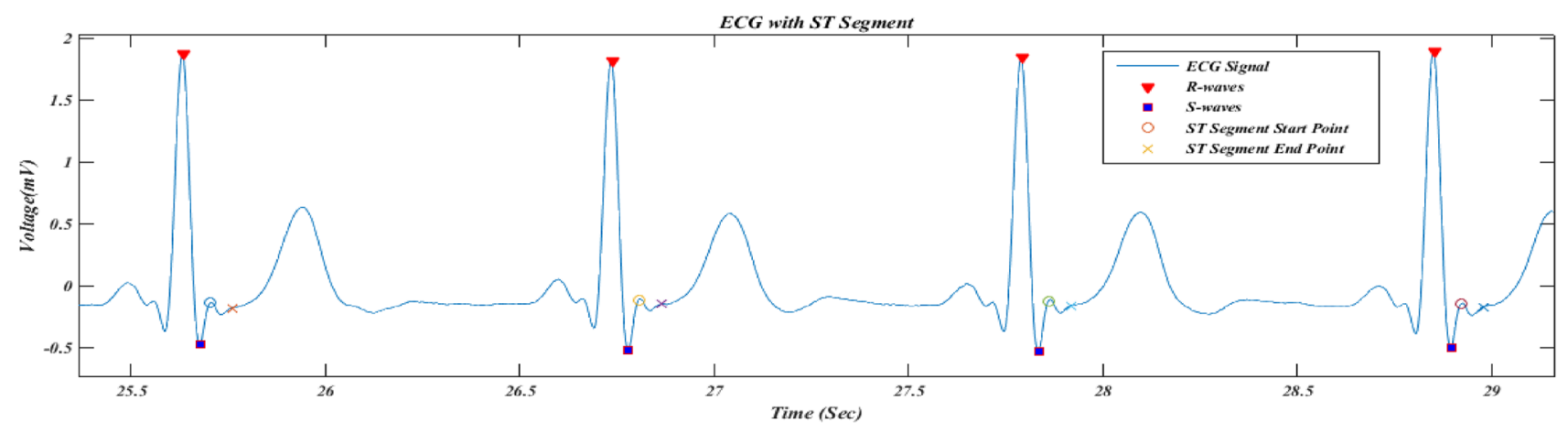

(a)

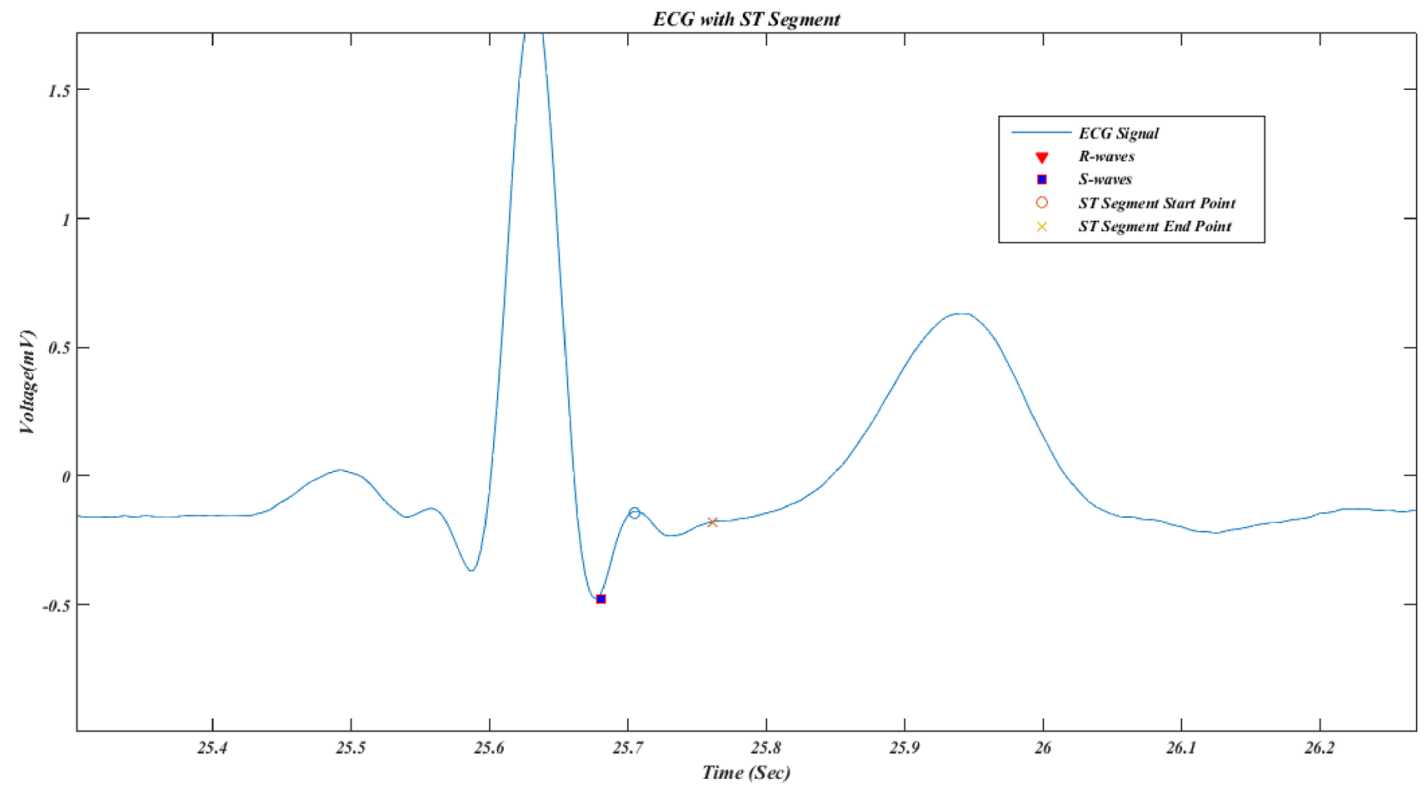

(b)

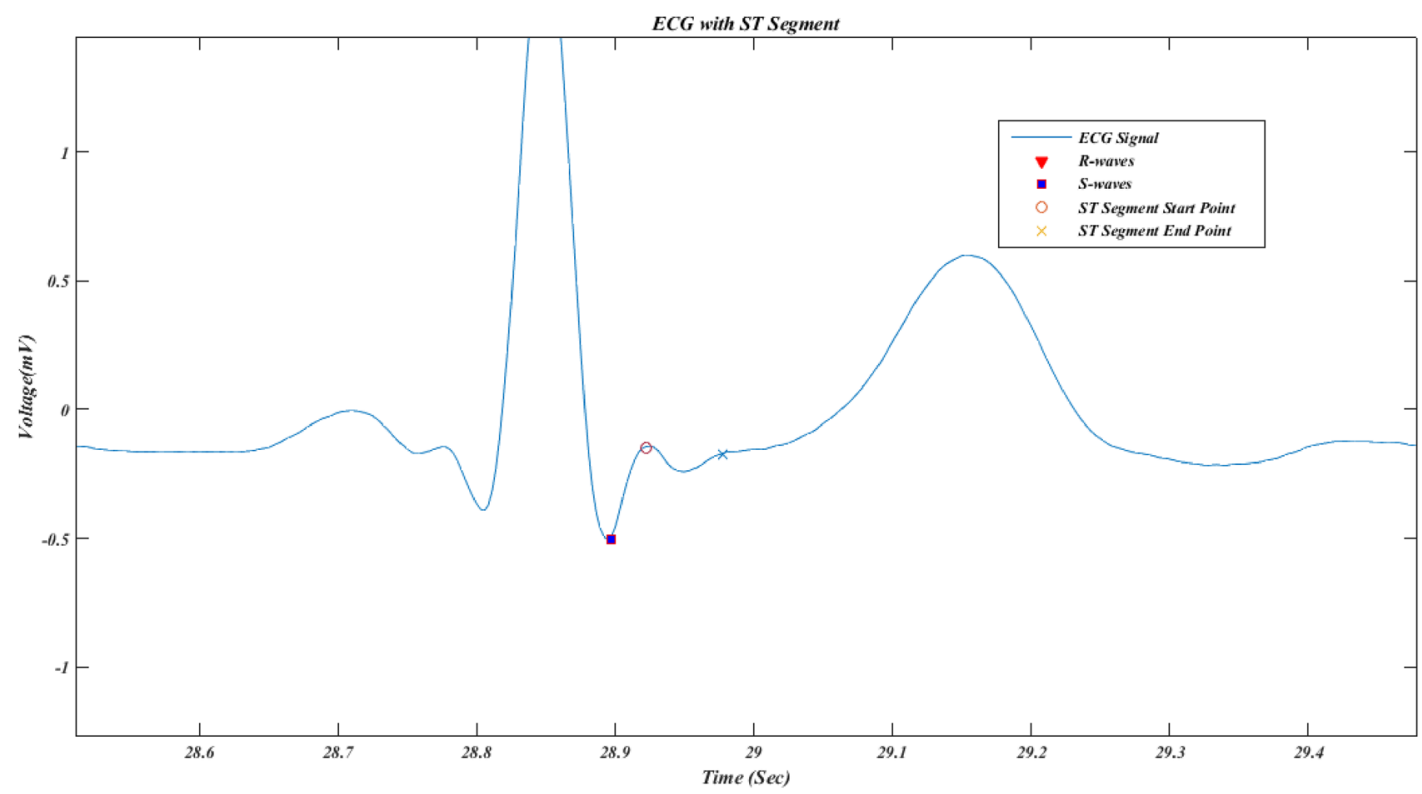

(c)

Fig. 12 Concave ST segment

\section{SN Applied Sciences}

a SPRINGER NATURE journal 


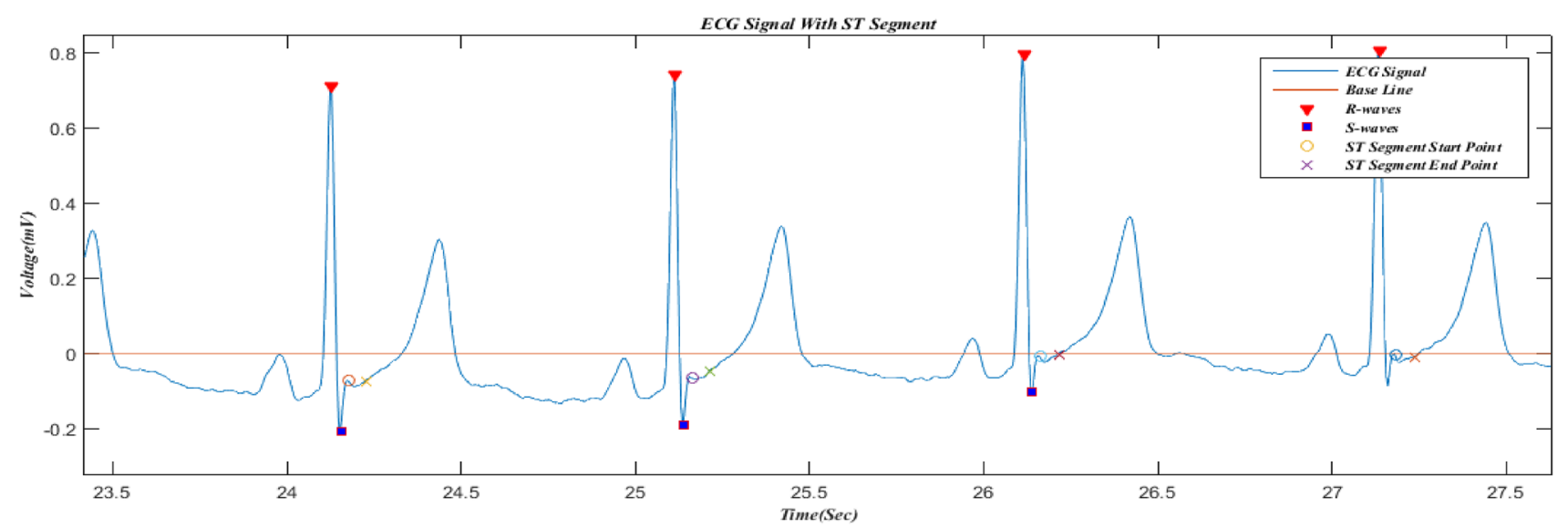

(d)

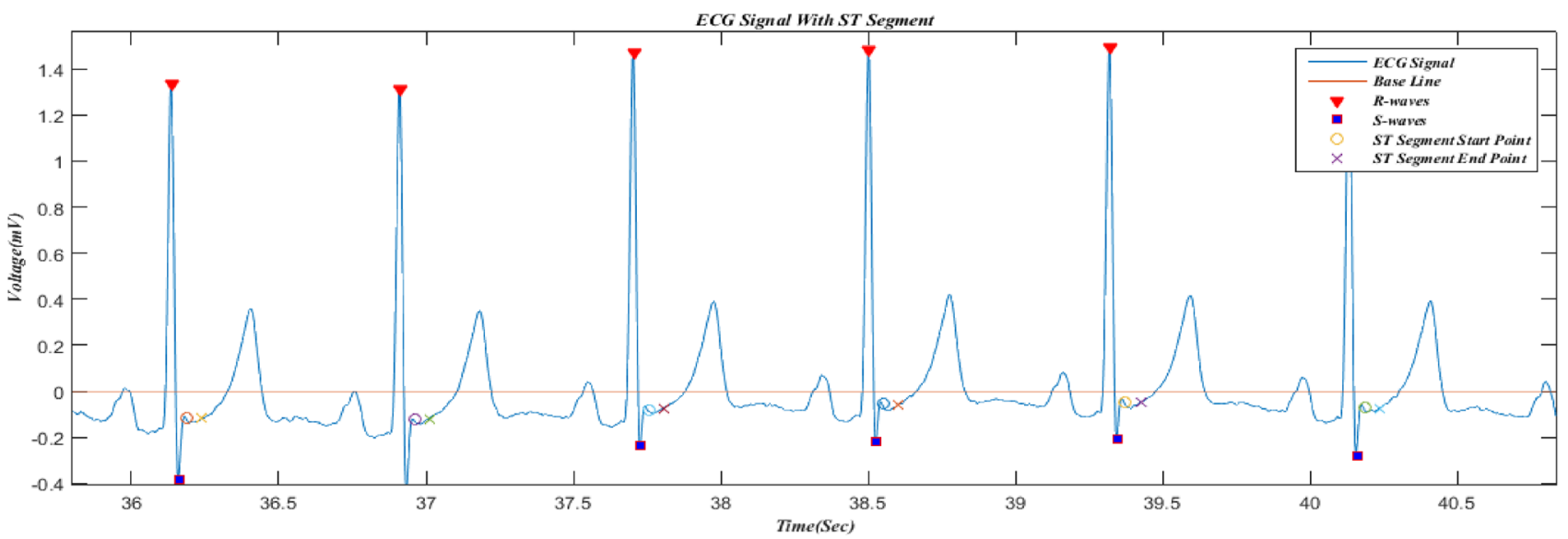

(e)

Fig. 12 (continued)

pattern by $R+x$ method [21]. In absence of $R$ wave or low amplitude of $R$ wave we have used Eq. (6). Thus, J point detection is more accurate than the other existing method [30]. On the other hand, for detecting ST segment end point most of the methods used time domain value were 5-60 ms counting from J point [28-30]. But in our proposed method we have used $\mathrm{T}$ wave pattern for detecting T wave start point that is, ST segment end point. Here we have used T wave slope value for detecting $T$ wave start point. Thus, ST segment detection is totally new method from the existing other methods $[27,28]$.

In addition, in previous study [27-30] ST segment is classified based on their amplitude value in $m V$ or $\mu V$. In our proposed method we used cross-correlation process where ST segment amplitude value is not mandatory. Another study [27] classified the ST segment based on their average amplitude value from base line of ECG signal. But in our proposed method base line is important only for detrending ECG signal not for classify the ST Segment.
Sometimes convex type ST segment is found under the baseline. That time amplitude-based ST segment classification is not work properly. In this case our proposed method works correctly. Previous works [27-30] use short term ECG signal most of them are less than 20 min. But in our proposed method we used minimum 30-min durations ECG signal and maximum 1-h episode. In this case, our proposed method is also applicable for long term ECG signal.

From the Tables 1 and 2 we have got the charts (Fig. 17) and (Fig. 18). Here Figs. 17 and 18 shows the bar chart of data classification accuracy. ST segment classified into five categories where we used two types of databases MIT-BIH ST change database and European ST-T change database. In Fig. 17, it shows the correctly identification of ST Segment in MIT-BIH ST change database. That means Concave, Convex, Up Slope, Down Slope and Horizontal type ST segment correctly selection accuracy bar-chart is given in Fig. 17. On the other hand, in Fig. 18 shows the bar chart 


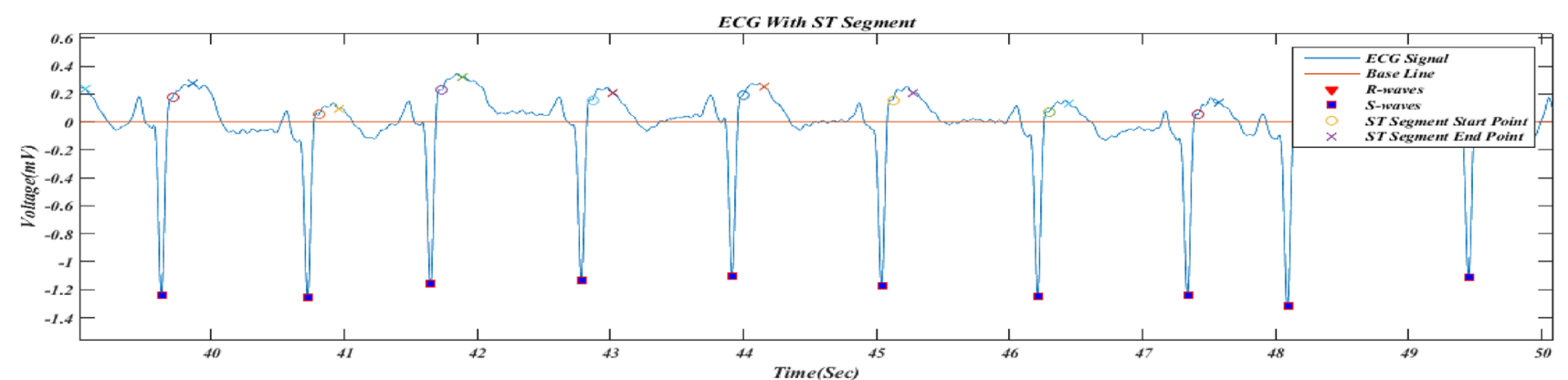

(a)

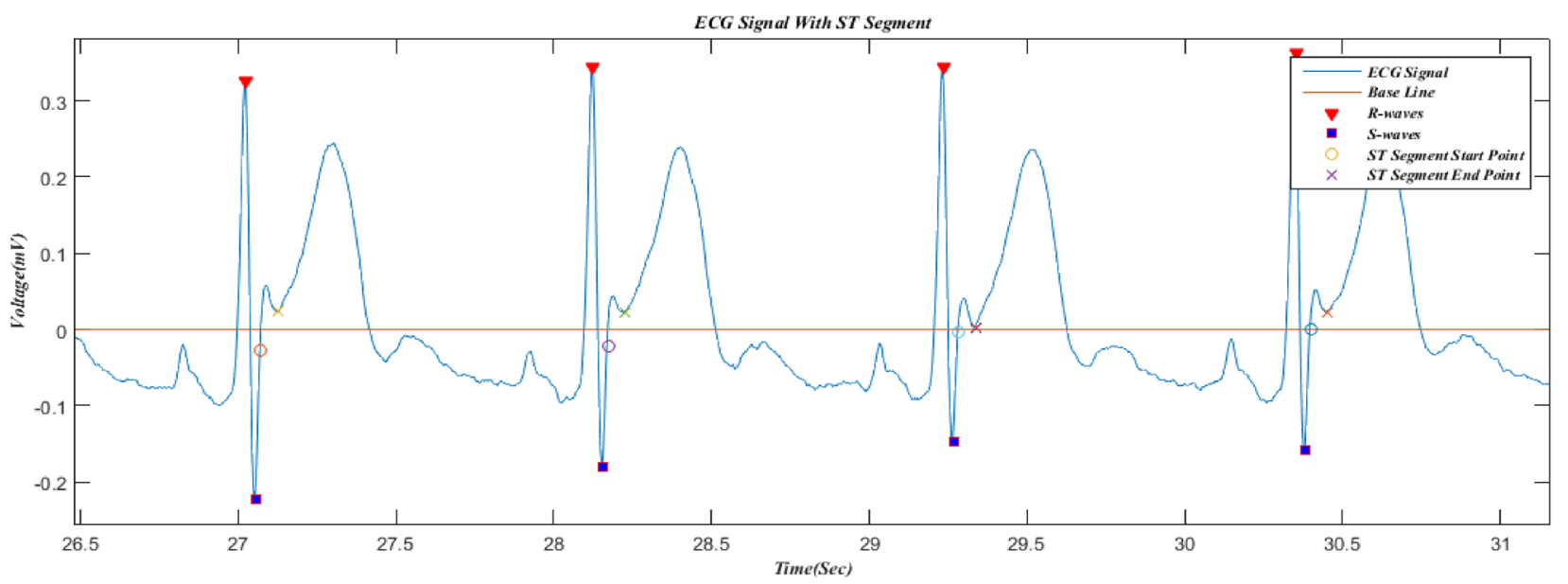

(b)

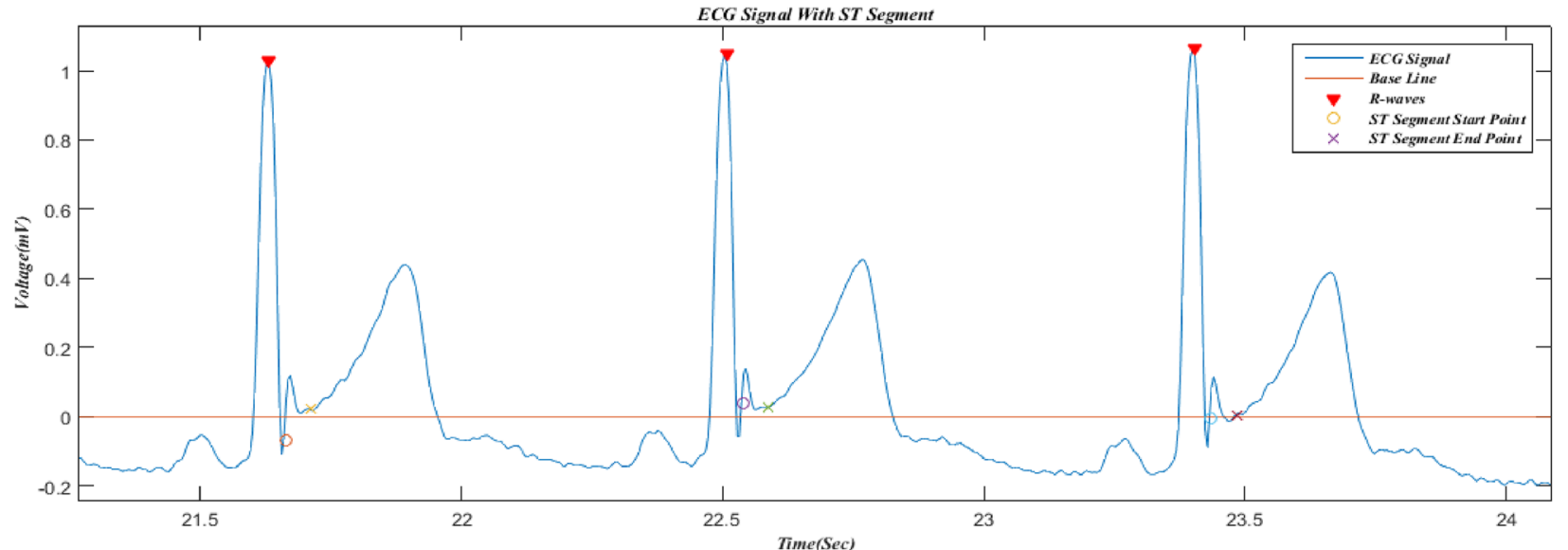

(c)

Fig. 13 Convex ST segment

of correctly selection accuracy of European ST-T change database. Comparing between both chart European ST-T change database ST segment classification accuracy more better than MIT-BIH ST change database.

Analysing the tables and charts we can say that overall accuracy of ST segment classification is more accurate in European ST-T change database than MIT-BIH ST change database. Most of the method provides ST shape elevation and depression [10] but does not provide any classification. This method [10] provides only $R$ wave-based process. Our proposed method correctly classifies the ST segment in absence of $R$ wave [22]. While automatic detecting, $R$ wave may be absent. In absence of $R$ wave, we have selected $\mathrm{S}$ wave for detecting ST segment. So, 


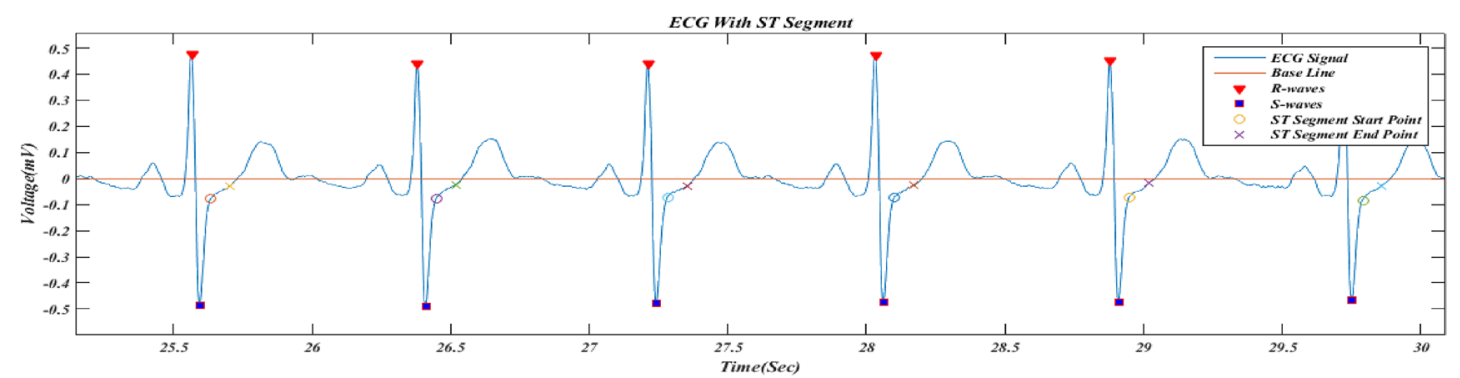

(a)

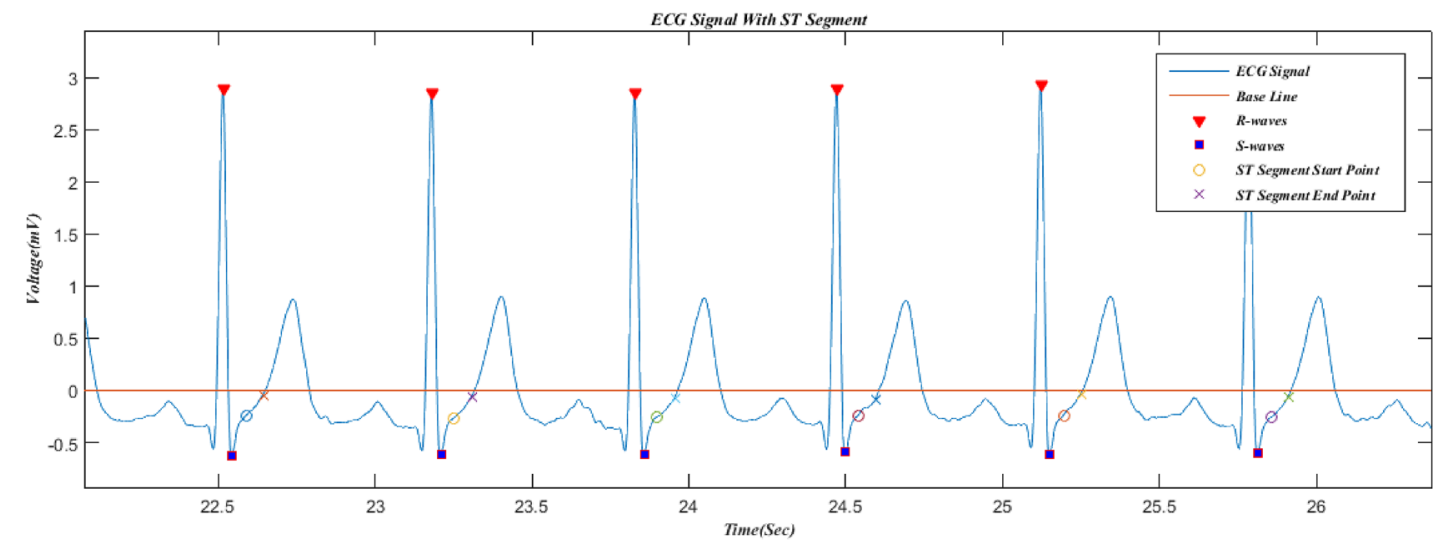

(b)

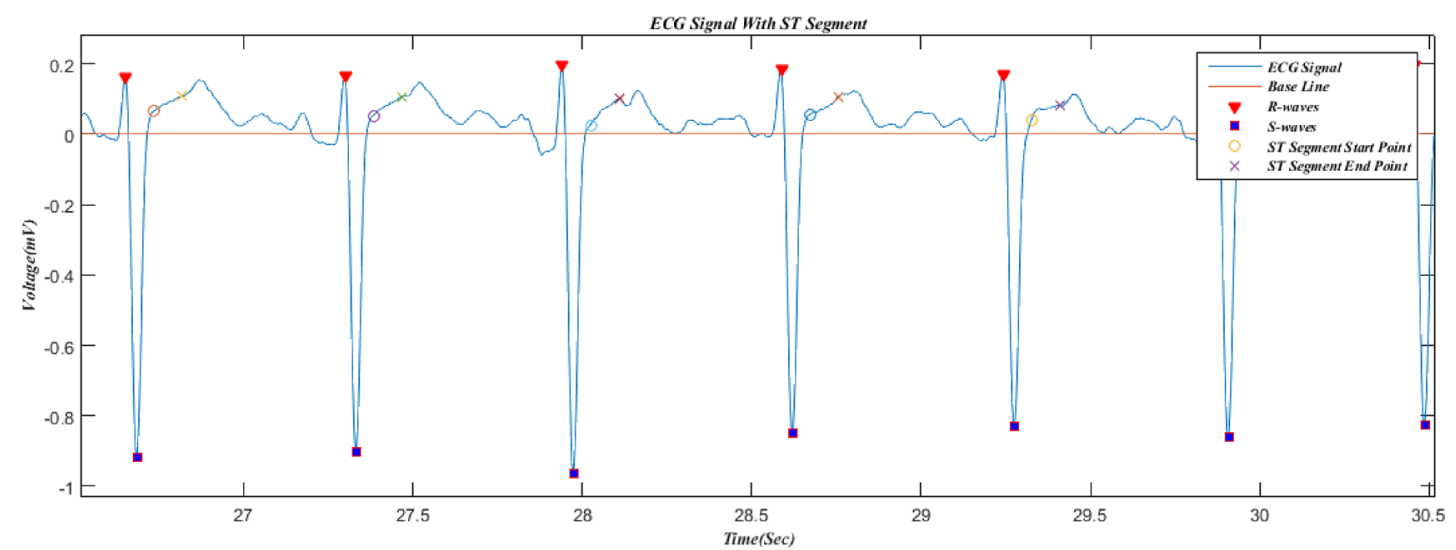

(c)

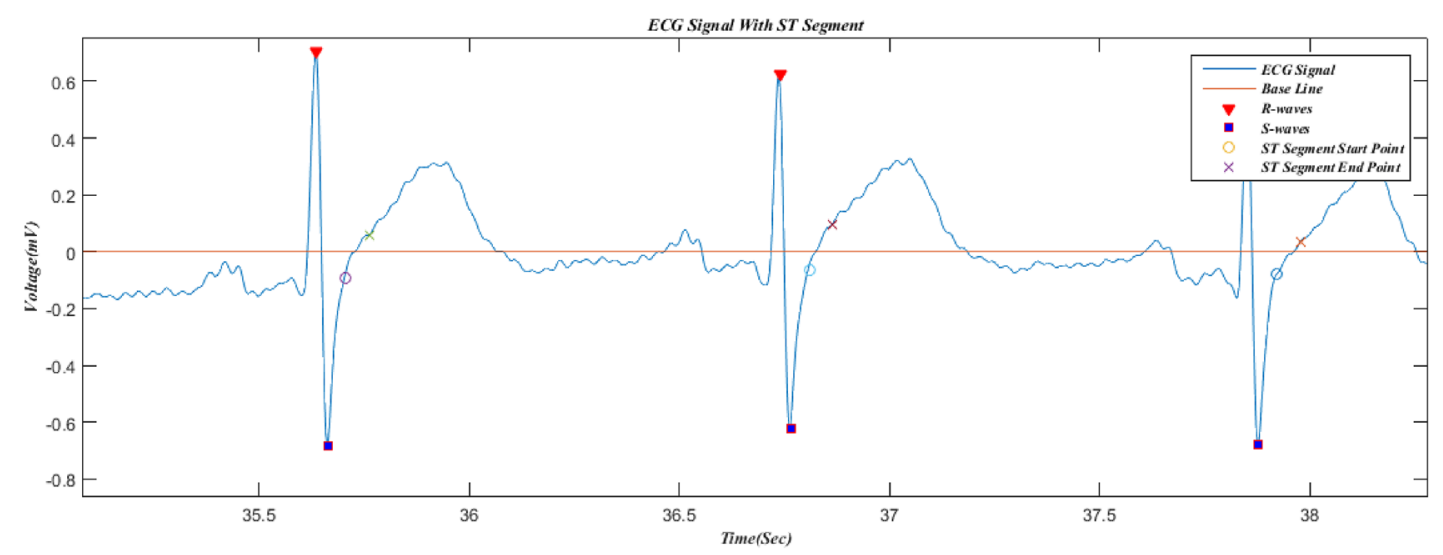

(d)

Fig. 14 Up slop ST segment 


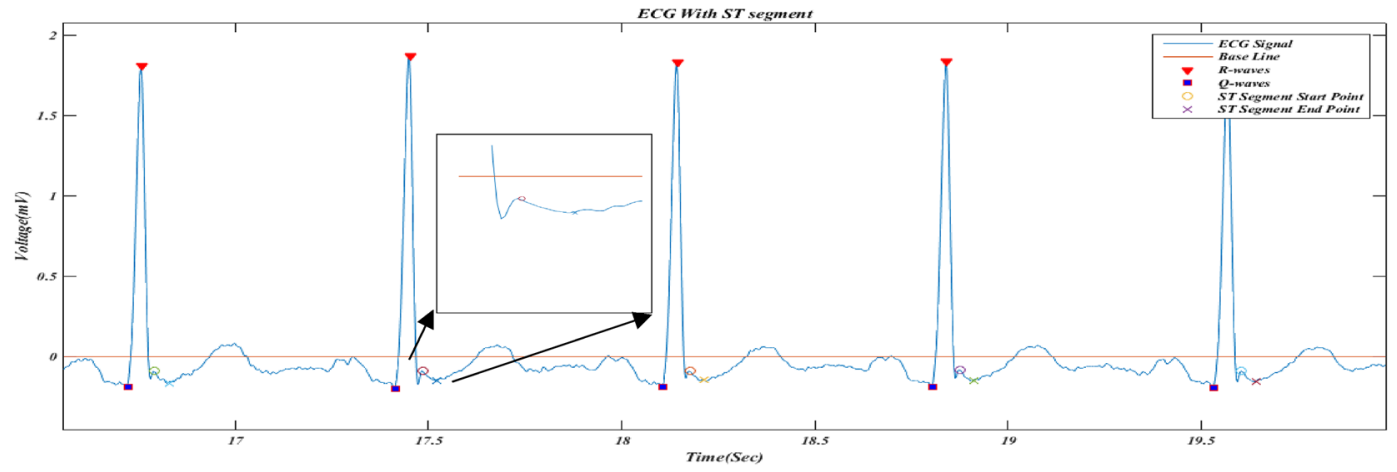

(a)

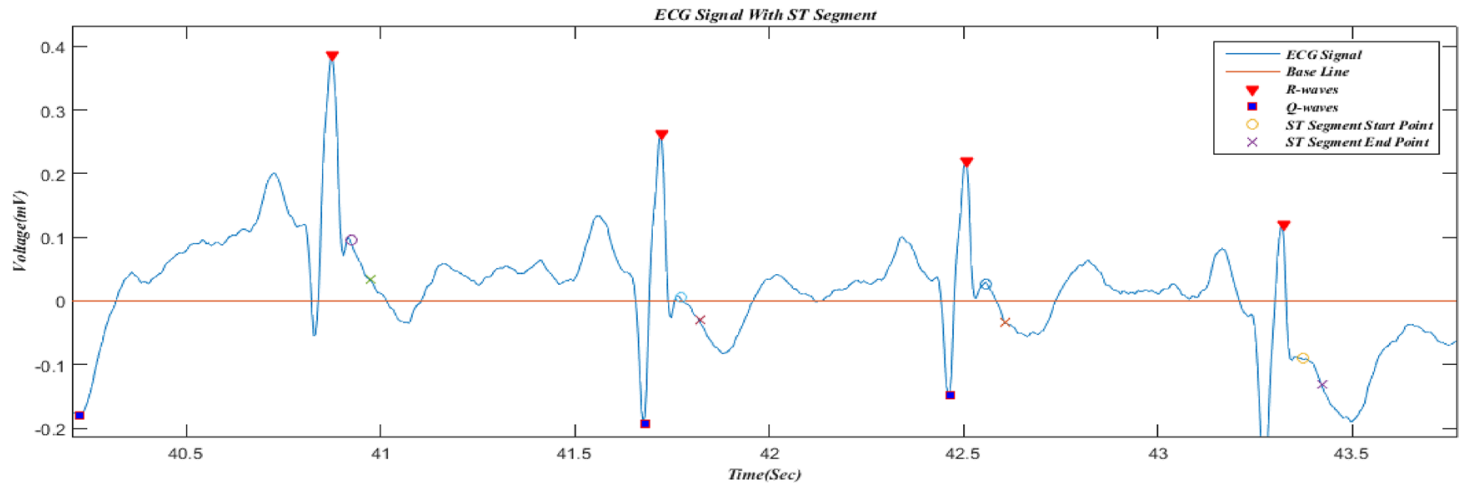

(b)

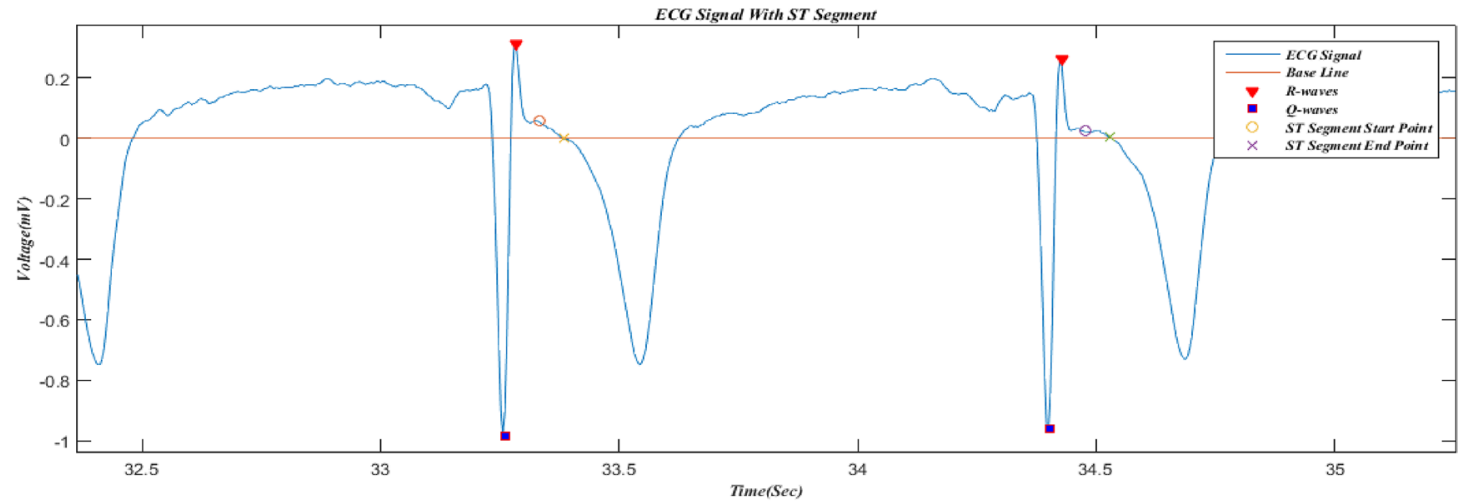

(c)

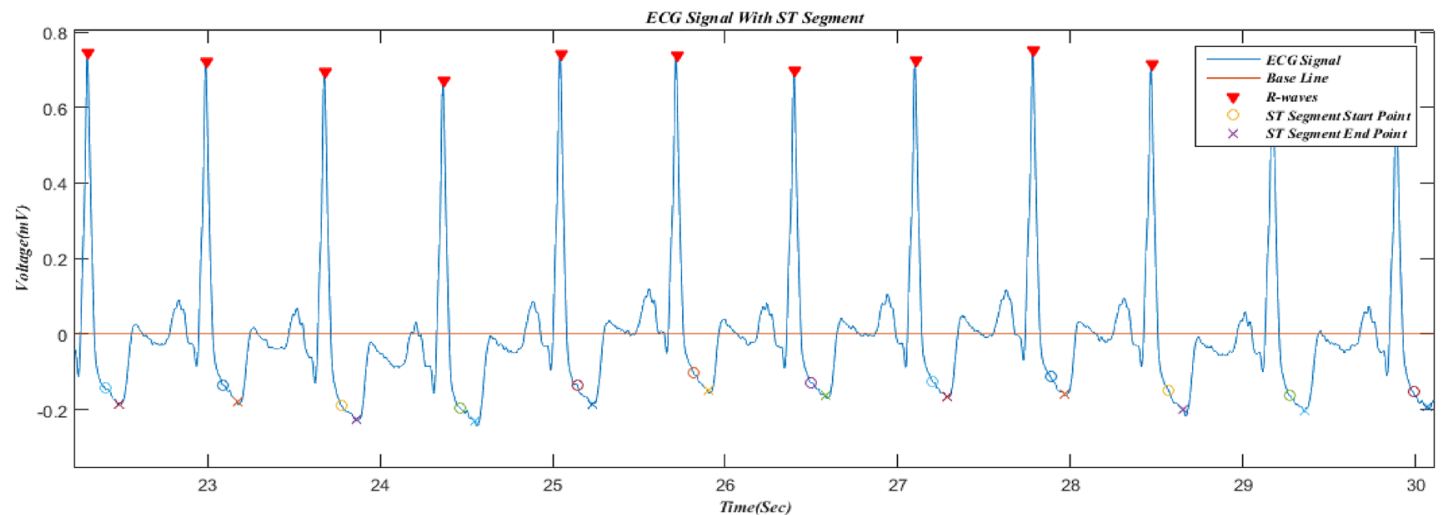

(d)

Fig. 15 Down slop ST segment

\section{SN Applied Sciences}

A SPRINGER NATURE journal 


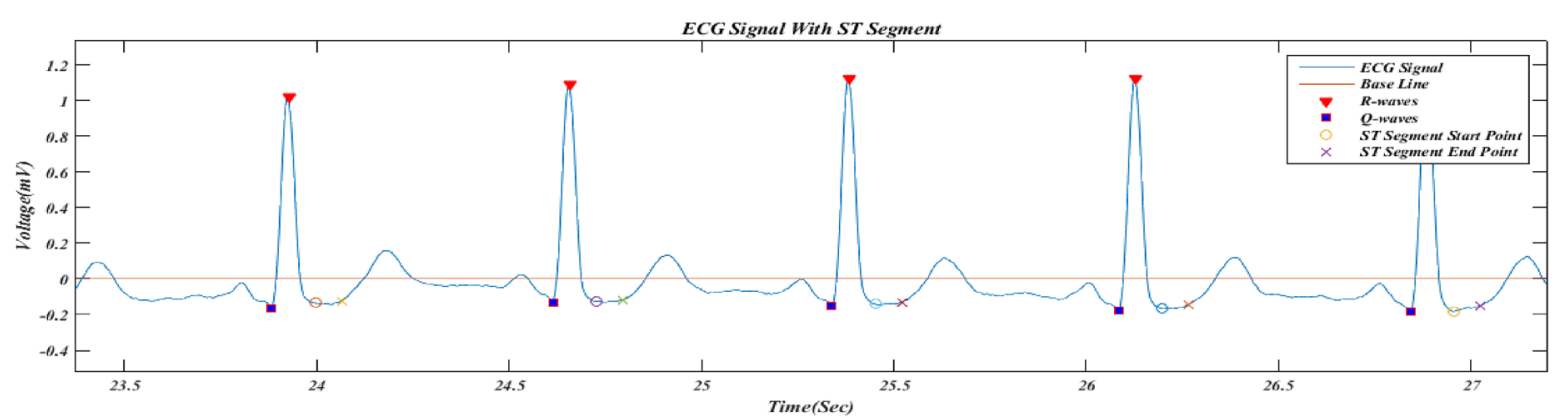

(a)

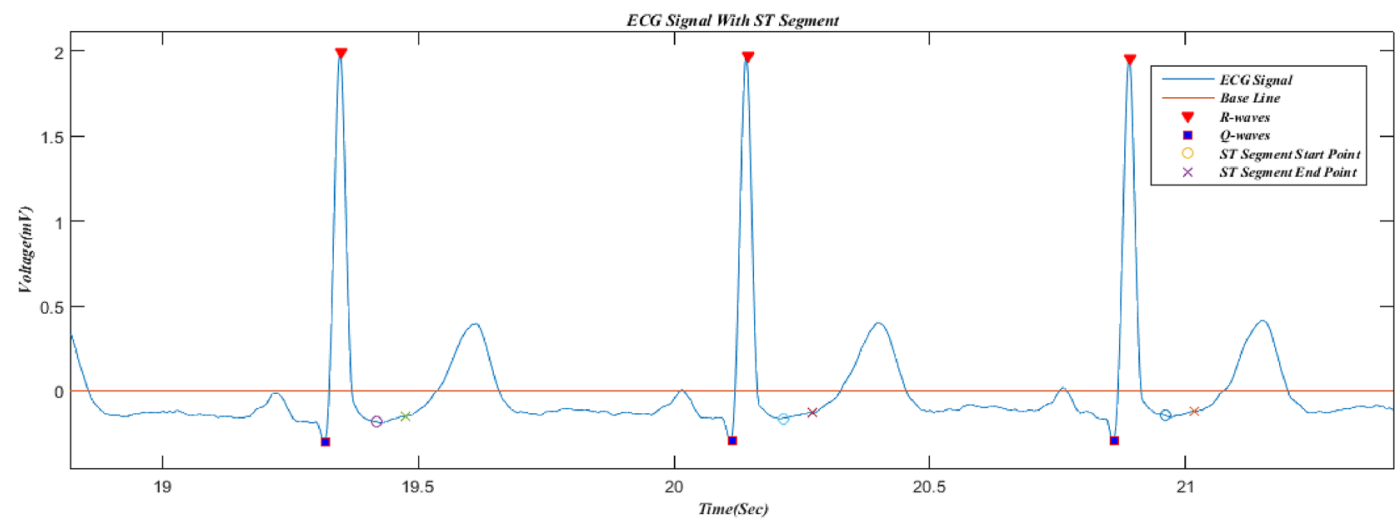

(b)

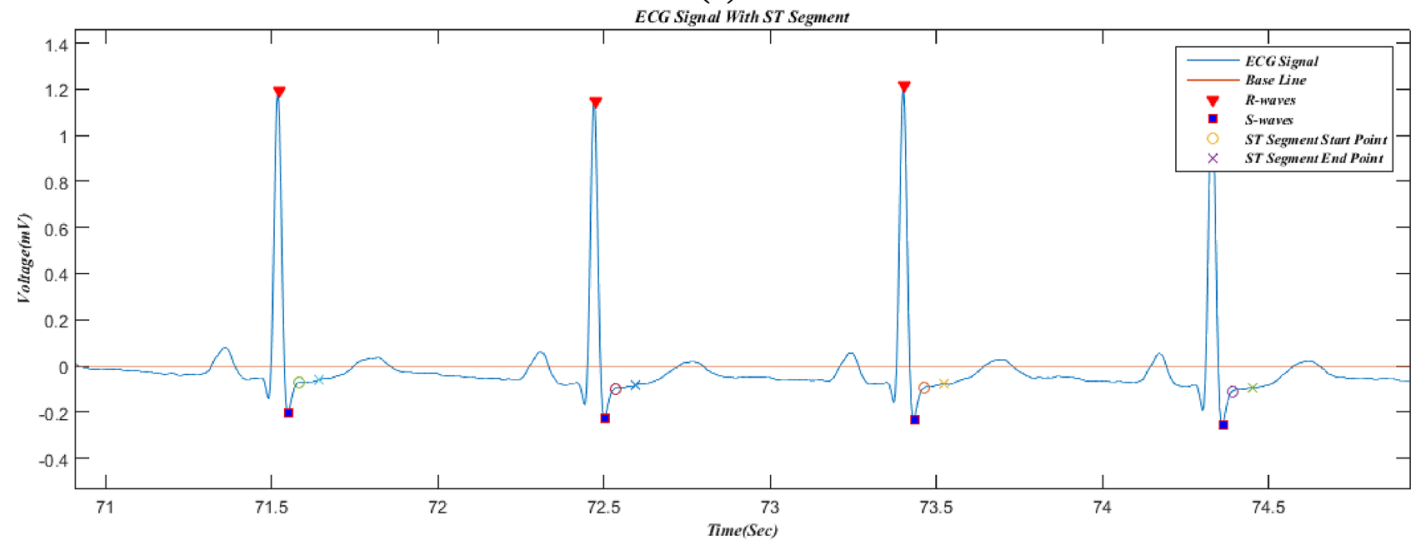

(c)

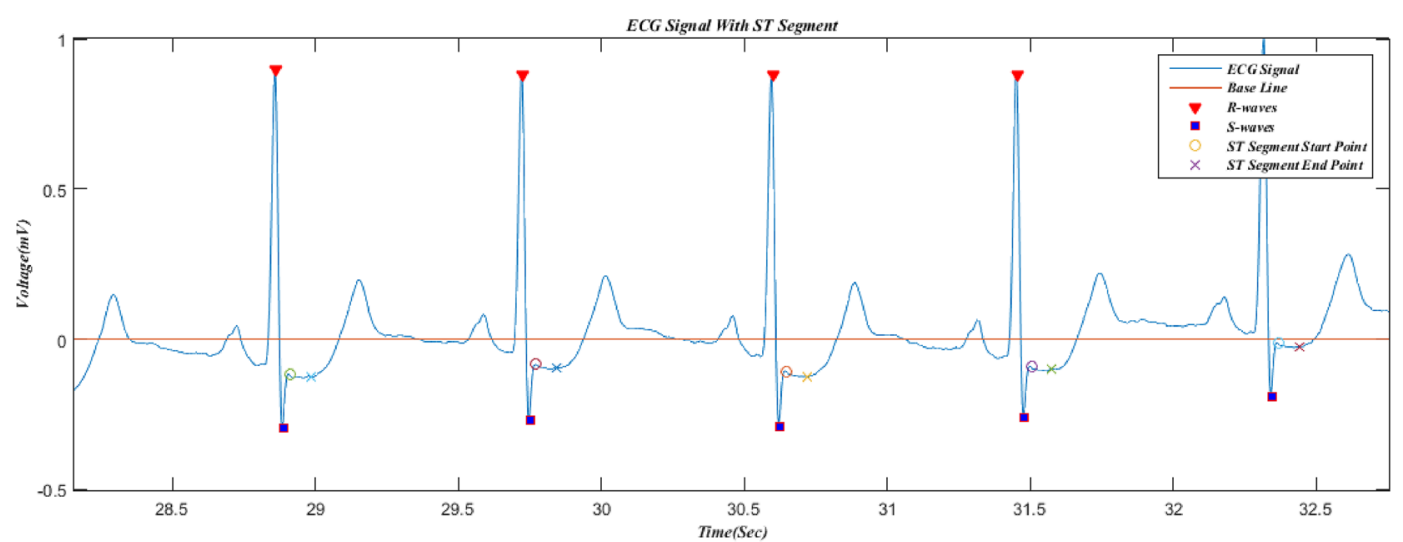

(d)

Fig. 16 Horizontal ST segment 
Table 1 ST segment selection table

\begin{tabular}{|c|c|c|c|c|c|c|c|}
\hline Database name & $\begin{array}{l}\text { Total } \\
\text { number of } \\
\text { beats }\end{array}$ & $\begin{array}{l}\text { Total number of } \\
\text { corrected beats }\end{array}$ & $\begin{array}{l}\text { Correctly } \\
\text { selected ST } \\
\text { segment }\end{array}$ & $\begin{array}{l}\text { Wrongly } \\
\text { selected ST } \\
\text { segment }\end{array}$ & $\begin{array}{l}\text { Correctly classi- } \\
\text { fied ST segment }\end{array}$ & $\begin{array}{l}\text { Wrongly } \\
\text { classified ST } \\
\text { segment }\end{array}$ & $\begin{array}{l}\text { Percentage of ST } \\
\text { segment correction } \\
(\%)\end{array}$ \\
\hline $\begin{array}{l}\text { Training data } \\
\text { (MIT-BIH ST } \\
\text { change data- } \\
\text { base) }\end{array}$ & 60,270 & 58,331 & 57,026 & 1305 & 56,434 & 592 & 98.96 \\
\hline $\begin{array}{l}\text { Testing data } \\
\text { (European } \\
\text { ST-T change } \\
\text { database) }\end{array}$ & 74,609 & 72,826 & 72,502 & 324 & 70,174 & 2328 & 96.79 \\
\hline
\end{tabular}

Table 2 ST segment classification accuracy

\begin{tabular}{llllll}
\hline Data & Concave (\%) & Convex (\%) & Up slope (\%) & Down slope (\%) & Horizontal (\%) \\
\hline Training data (MIT-BIH ST change) & 98.77 & 97.47 & 90.35 & 85.03 & 69.42 \\
$\begin{array}{l}\text { Testing data (European ST-T change } \\
\text { database) }\end{array}$ & 95.58 & 95.92 & 97.86 & 95.18 & 96.36 \\
\hline
\end{tabular}

\section{MIT-BIH ST change Database}

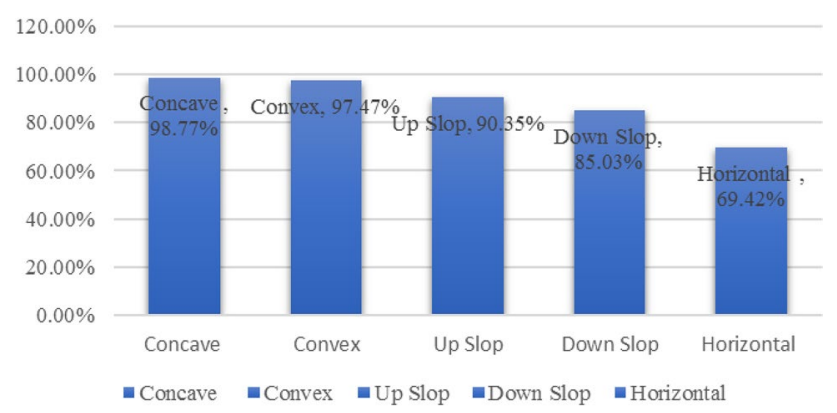

Fig. 17 ST segment classification accuracy chart for MIT-BIH ST change database we can easily identify the ST segment. Overall accuracy of selecting ST segment is $97.87 \%$.

Long-term ECG contains trend which may be linear or non-linear. After detrending this we can easily identify the base line and quality of ECG. Our proposed method based on Cross-Correlation process provides $92.1 \%$ accuracy where we have identified 3331 concave shape out of $3485,13,414$ convex shape out of $13,984,25,362$ horizontal shape out of 26,318, 1265 downslope shape out of 1329 and 26,802 up slopes shape out of 27,386 . We have used total 47 database which contain total 1, 34,879 beats. Our proposed method exhibits more accuracy than other existing methods [27-30].

For clinical diagnosis, our proposed method will be more helpful in myocardial ischemia and ST segment monitoring. This method will also help the physicians to easily identify the different types of ST shapes in the ECG
Fig. 18 ST segment classification accuracy chart for European ST-T change database

\section{European ST-T change database}

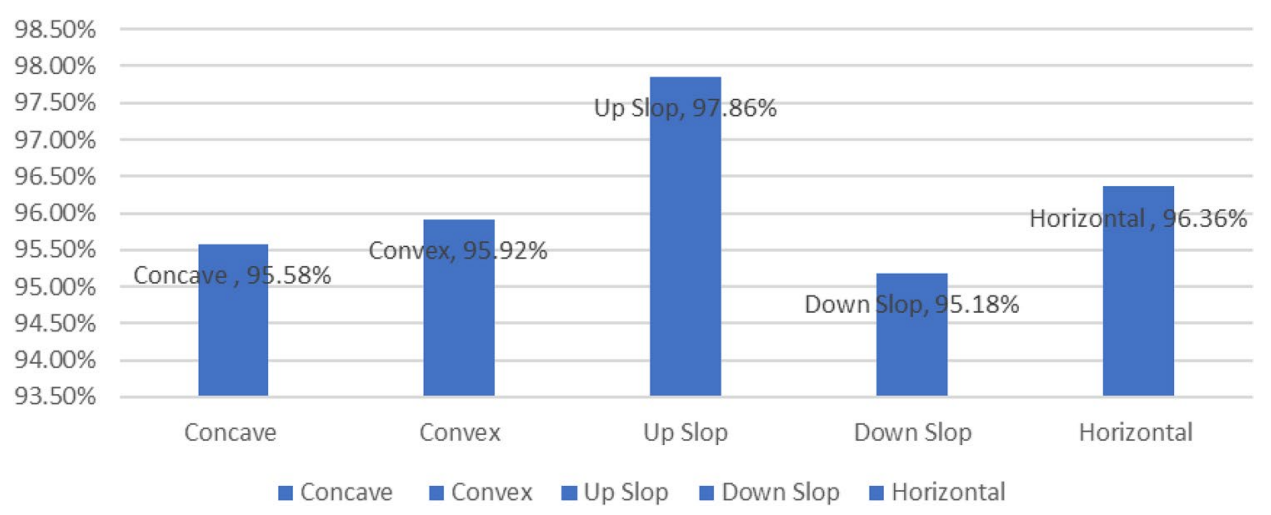


signal. Our proposed method has verified all 12 lead ECG signal and their performance accuracy is $92.1 \%$. We hope it will enrich the craniological research also.

Though we get $92.1 \%$ accuracy for ST segment classification but there are some drawbacks and limitations. For classifying ST Segment ECG data pre-processing must be needed. Without ECG data pre-processing ST Segment cannot be detect correctly. For low amplitude of $\mathrm{R}$ wave and S wave it is too difficult to detect ST segment. For long term ECG signal our proposed method takes time to evaluate.

\section{Conclusion}

Cross-Correlation process is used for the first time to classify ST segments. That is our main contribution. On the other hand, we used the Right Triangle hypotenuse system for detecting ST segment end point. We also used long length ECG signal of minimum 30 min or above. As a result, our proposed method is totally new for classifying ST segment in the ECG signal. Selection and classification of ST segment is very important for detecting myocardial ischemia. Total Twenty-Eight MIT-BIH ST change databases, Nineteen European ST-T change database are used, and 5 supervised signals are selected for comparing with signal shape. We have used total 134,879 beats where 58,331 beats from MIT BIH database and 74,609 beats from European ST-T database. We have correctly selected total 126,608 ST segment. ST segment classification accuracy is $88.02 \%$ for MIT BIH data base and $96.18 \%$ for European ST-T database. Overall accuracy for all database is $92.1 \%$. Using this method, we can easily identify the ST segment shape using cross correlation. We hope it will be more and more helpful in clinical diagnosis for detecting major heart diseases like Myocardial Ischemia hence will reduce the number of deaths occurring all through the year in the whole world.

\section{Compliance with ethical standards}

Conflict of interest On behalf of all authors, the corresponding author states that there is no conflict of interest.

\section{References}

1. Van Oosterom A (2009) Measuring the T wave of the electrocardiogram; the how and why. Meas Sci Rev 9(3):53-63

2. Hadjem M, Naït-Abdesselam F (2015) An ECG T-wave anomalies detection using a lightweight classification model for wireless body sensors. In: 2015 IEEE international conference on communication workshop (ICCW). IEEE. 2015 June, pp 278-283
3. Gajendra E, Kumar MJ (2015) A novel approach of ECG classification for diagnosis of heart diseases. Int J Adv Res Comput Eng Technol (IJARCET) 4(11):4096-4100

4. Sarkaleh MK, Shahbahrami A (2012) Classification of ECG arrhythmias using discrete wavelet transform and neural networks. Int J Comput Sci Eng Appl 2(1):1

5. García J, Sornmo L, Olmos S, Laguna P (2000) Automatic detection of ST-T complex changes on the ECG using filtered RMS difference series: application to ambulatory ischemia monitoring. IEEE Trans Biomed Eng 47(9):1195-1201

6. Haeberlin A, Studer E, Niederhauser T, Stoller M, Marisa T, Goette J, Jacomet M, Traupe T, Seiler C, Vogel R (2014) Electrocardiographic ST-segment monitoring during controlled occlusion of coronary arteries. J Electrocardiol 47(1):29-37

7. Sandau KE, Smith M (2009) Continuous ST-segment monitoring: 3 case studies in progressive care. Crit Care Nurse 29(5):18-27

8. Wang H, Hollingsworth J, Mahler S, Arnold T (2010) Diffuse ST segment depression from hypothermia. Int J Emerg Med 3(4):451-454

9. Belfort MA, Saade GR, Thom E, Blackwell SC, Reddy UM, Thorp JM Jr, Tita AT, Miller RS, Peaceman AM, McKenna DS, Chien EK (2015) A randomized trial of intrapartum fetal ECG ST-segment analysis. N Engl J Med 373(7):632-641

10. Bulusu SC, Faezipour M, Ng V, Nourani M, Tamil LS, Banerjee S (2011) Transient ST-segment episode detection for ECG beat classification. In: Life science systems and applications workshop (LiSSA), 2011 IEEE/NIH, April 2011, pp 121-124

11. Prasad ST, Varadarajan S (2015) Analysis of ST segment abnormalities in ECG using signal block averaging technique. Int J Adv Res Comput Commun Eng 4(2):163-167

12. Zeng R, Diao K, Cheng F, Ma S (2016) ST Segment. In: Zeng R (ed) Graphics-sequenced interpretation of ECG. Springer, Singapore

13. http://physionet.org/cgi-bin/atm/ATM?database=ptbdbandto ol=plot_waveforms

14. Narsimha B, Suresh E, Punnamchandar K, Reddy MS (2011) Denoising and QRS detection of ECG signals using Empirical Mode Decomposition. In: 2011 international conference on communications and signal processing (ICCSP). IEEE, Feb 2011, pp 439-442

15. Blanco-Velasco M, Weng B, Barner KE (2008) ECG signal denoising and baseline wander correction based on the empirical mode decomposition. Comput Biol Med 38(1):1-13

16. Zhidong Z, Chan M (2008) A novel cancellation method of powerline interference in ECG signal based on EMD and adaptive filter. In: 11 th IEEE international conference on communication technology, 2008. ICCT 2008. IEEE. Nov 2008, pp 517-520

17. Nimunkar AJ, Tompkins WJ (2007) R-peak detection and signal averaging for simulated stress ECG using EMD. In: 29th annual international conference of the IEEE, engineering in medicine and biology society, 2007. EMBS 2007. IEEE, Aug 2007, pp $1261-1264$

18. Barill T (2003) An ECG primer. Nursecom Educational Technologies. https://www.co.grand.co.us/DocumentCenter/View/626/ ECG-Primer-The-Six-Second-ECG-Guidebook-Nursecom-Educa tional-Technologies

19. London MJ, Hollenberg M, Wong MG, Levenson L, Tubau JF, Browner W, Mangano DT (1988) Intraoperative myocardial ischemia: localization by continuous 12-lead electrocardiography. Anesthesiology 69(2):232-241

20. Junttila MJ, Sager SJ, Tikkanen JT, Anttonen O, Huikuri HV, Myerburg RJ (2012) Clinical significance of variants of J-points and J-waves: early repolarization patterns and risk. Eur Heart J 33(21):2639-2643

21. Suzuki Y, Ono K (1992) Personal computer system for ECG STsegment recognition based on neural networks. Med Biol Eng Compu 30(1):2-8 
22. Jekova I, Krasteva V, Leber R, Schmid R, Twerenbold R, Reichlin T, Müller C, Abächerli R (2017) A real-time quality monitoring system for optimal recording of 12-lead resting ECG. Biomed Signal Process Control 34:126-133

23. Zhang Q, Manriquez Al, Médigue C, Papelier Y, Sorine M (2006) An algorithm for robust and efficient location of T-wave ends in electrocardiograms. IEEE Trans Biomed Eng 53(12):2544-2552

24. Shuo Y, Desong B (2008) Automatic detection of $t$-wave end in ECG signals. In: Second international symposium on intelligent information technology application, 2008. IITA'08. IEEE. Dec 2008, vol 3, pp 283-287

25. Oosterom AV (2003) The dominant T wave and its significance. J Cardiovasc Electrophysiol 14:S180-S187

26. Yochum M, Renaud C, Jacquir S (2016) Automatic detection of $\mathrm{P}, \mathrm{QRS}$ and T patterns in 12 leads ECG signal based on CWT. Biomed Signal Process Control 25:46-52

27. Xu M, Wei S, Qin X, Zhang Y, Liu C (2015) Rule-based method for morphological classification of ST segment in ECG signals. J Med Biol Eng 35(6):816-823
28. Veldkamp RF, Sawchak S, Pope JE, Califf RM, Krucoff MW (1996) Performance of an automated real-time ST-segment analysis program to detect coronary occlusion and reperfusion. J Electrocardiol 29(4):257-263

29. Hu F, Li C, Zhang Y, Jin D, Ma Z, Sun Y, Wang J (2015) A morphological classification method of ECG ST-segment based on curvature scale space. J Biosci Med 3(09):38

30. www.medical.philips.com. 452296220161 DEC 2006. https:// www.mc.vanderbilt.edu/documents/7north/files/STSegmentM onitoring\%204522\%20962\%2020161.pdf

Publisher's Note Springer Nature remains neutral with regard to jurisdictional claims in published maps and institutional affiliations. 